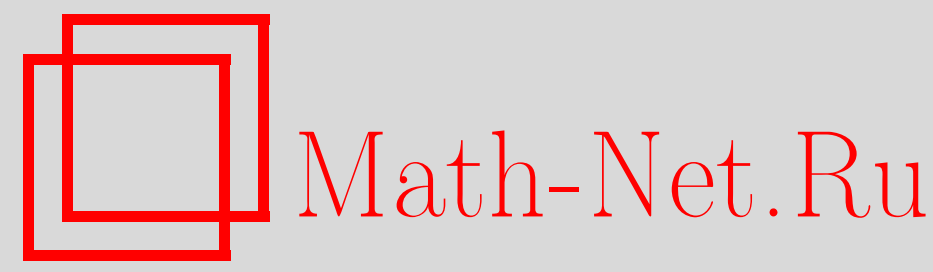

Ю. М. Дюкарев, О неопределенности интерполяционных задач в классе Стилтьеса, Матем. сб., 2005, том 196, номер 3, 61-88

DOI: https://doi.org/10.4213/sm1276

Использование Общероссийского математического портала Math-Net.Ru подразумевает, что вы прочитали и согласны с пользовательским соглашением

http://www . mathnet.ru/rus/agreement

Параметры загрузки:

IP : 107.22 .136 .117

26 апреля 2023 г., 09:59:10 
УДК 517.5

\author{
Ю.М. Дюкарев
}

\title{
О неопределенности интерполяционных задач в классе Стилтьеса
}

\begin{abstract}
В статье введено понятие упорядоченных семейств интерполяционных задач в классе Стилтьеса. С помощью упорядоченных семейств введено понятие предельной интерполяционной задачи в классе Стилтьеса. Доказано, что у предельной интерполяционной задачи существует решение. Для предельной интерполяционной задачи в классе Стилтьеса получен критерий полной неопределенности. Во вполне неопределенном случае дано описание всех решений в терминах дробно-линейных преобразований. Общие построения проиллюстрированы на примерах проблемы моментов Стилтьеса и задачи Неванлинны-Пика в классе Стилтьеса.

Библиография: 21 название.
\end{abstract}

\section{Введение}

В последние два десятилетия в статьях многих авторов были предложены общие подходы к решению различных интерполяционных проблем типа задачи Неванлинны-Пика, задачи Каратеодори, проблем моментов Гамбургера и Стилтьеса и т.д. В этих работах можно выделить два основных направления исследований: интерполяция в классе неванлинновских функций и интерполяция в классе стилтьесовских функций. В том и другом случаях рассматривают близкие по постановкам задачи, но методы их решения существенно различаются. Общие подходы к теории интерполяционных задач для неванлинновских функций были предложены в статьях [1]-[5], а для стилтьесовских функций-в статьях [6]-[8]. В этой статье мы будем рассматривать только интерполяционные задачи для стилтьесовских функций.

В процитированных выше работах интерполяционные задачи решались так, чтобы сразу оказались выполненньми все интерполяционные условия. Однако многие интерполяционные задачи нельзя решить непосредственно по такой схеме. Примерами являются проблема моментов Стилтьеса с бесконечным числом заданных моментов, задача Неванлинны-Пика для стилтьесовских функций с бесконечным числом узлов интерполяции и им подобные проблемы. Именно по этой причине в статьях [6]-[8] для дискретного случая рассматривались только интерполяционные задачи с конечным числом узлов интерполяции.

В классической теории дискретные интерполяционные задачи с бесконечным числом узлов интерполяции (см. [9]-[11]) решались по следующей схеме. Сначала решается усеченная интерполяционная задача, в которой должны вьполняться лишь первые $n$ интерполящионных условий. После этого, устремляя $n$ к бесконечности, рассматриваем интерполяционную задачу с бесконечным числом узлов интерполяции, которую естественно назвать предельной интерполяционной задачей.

(C) Ю. М. ДЮКАРЕв 2005 
При этом удается выяснить условия неопределенности задачи с бесконечньм числом узлов интерполяции и дать описание множества всех решений для неопределенного случая. Следует подчеркнуть, что к настоящему времени не были предложены достаточно обшие подходы, являюшиеся обобшением изложенной идеи решения интерполяционных задач для неванлинновских или стилтьесовских функций.

В этой статье предложен общий подход к исследованию предельных интерполяционных задач для стилтьесовских оператор-функций (о.ф.), который содержит в себе все основные идеи метода перехода от усеченных интерполяционных задач к предельной интерполяционной задаче. При этом усеченные задачи могут иметь весьма сложную структуру, их совокупность не обязана быть счетным множеством, а две соседние задачи могут отличаться друг от друга произвольным множеством дополнительных интерполяционных условий. В статье используются многие идеи и методы подхода В.П. Потапова к решению интерполяционных задач [12]-[15].

И, заканчивая введение, перечислим основные новые понятия и результаты статьи.

В $§ 1$ приведена несколько модифицированная по сравнению с [6]-[8] обобщенная интерполяционная задача стилтьесовского типа. В таком виде интерполяционная задача лучше приспособлена для наших целей.

В $\S 2$ исследованы фундаментальные свойства главных решений обобщенной интерполяционной задачи (теорема 2) и вводятся операторные интервалы Вейля (определение 7). Эти интервалы при интерполяции в классе стилтьесовских функций являются аналогами кругов Вейля при интерполяции в классе неванлинновских функций.

В $\S 3$ вводится понятие унитарной эквивалентности двух обобщенных интерполяционных задач (определение 8) и доказано совпадение множеств решений унитарно эквивалентных задач (лемма 2). Далее вводится понятие упорядоченности двух интерполяционных задач стилтьесовского типа (определение 10) и доказывается естественная упорядоченность по включению соответствующих множеств решений и интервалов Вейля (теорема 3$)$.

В $\S 4$ вводятся понятия упорядоченного семейства обобщенных интерполяционных задач, предельной интерполяционной задачи и множества ее решений (определения 11, 12). Доказывается сушествование главњых решений предельной интерполяционной задачи (теорема 4) и вводится понятие предельных интервалов Вейля (определение 13).

В $\S 5$ доказьвается инвариантность рангов предельных интервалов Вейля (48), вводится понятие полной неопределенности предельной интерполяционной задачи (определение 14) и доказывается критерий полной неопределенности предельной интерполяционной задачи (теорема 5).

В $\S 6$ указана специальная нормировка (53) резольвентных матриц усеченных интерполяционных задач. При такой нормировке семейство резольвентных матриц усеченных интерполяционных задач во вполне неопределенном случае сходится к резольвентной матрице предельной интерполяционной задачи (теорема 6). Теорема 8 дает описание множества всех решений вполне неопределенной предельной интерполяционной задачи.

В $\S 7$ рассмотрены два примера: операторная задача Неванлинны-Пика и опе- 
раторная проблема моментов Стилтьеса. С помощью теоремы 5 для них получены критерии полной неопределенности и с помощью теоремы 8 дано описание всех решений соответствующих предельных интерполяционных задач во вполне неопределенном случае.

\section{§1. Обобщенная интерполяционная задача}

Введем обозначения: $\mathbb{R}_{+}=\{x \in \mathbb{R}: x \geqslant 0\}, \mathbb{R}_{-}=\{x \in \mathbb{R}: x<0\}$, $\mathbb{C}_{+}=\{z \in \mathbb{C}: \operatorname{Im} z>0\}, \mathbb{C}_{-}=\{z \in \mathbb{C}: \operatorname{Im} z<0\}, \mathbb{C}_{ \pm}=\mathbb{C}_{+} \cup \mathbb{C}_{-}$, $\Pi_{-}=\{z \in \mathbb{C}: \operatorname{Re} z<0\}$.

Пусть $\mathscr{G}_{1}, \mathscr{G}_{2}$ - сепарабельные и $\mathscr{H}$ - конечномерное гильбертовы пространства. Символом $\left\{\mathscr{G}_{1}, \mathscr{G}_{2}\right\}$ обозначим множество всех ограниченных линейных операторов, действующих из $\mathscr{G}_{1}$ в $\mathscr{G}_{2}$, символом $\left\{\mathscr{G}_{1}\right\}$ обозначим множество $\left\{\mathscr{G}_{1}, \mathscr{G}_{1}\right\}$, а символом $\left\{\mathscr{G}_{1}\right\}_{H}$ - множество ограниченных эрмитовых операторов в $\mathscr{G}_{1}$. Опера-

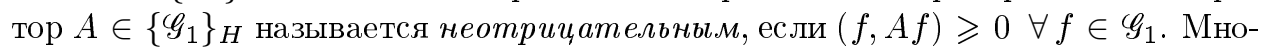
жество неотрицательных операторов в $\mathscr{G}_{1}$ обозначим символом $\left\{\mathscr{G}_{1}\right\} \geqslant$. Неотрицательный оператор $A \in\left\{\mathscr{G}_{1}\right\} \geqslant$ называется строго положсительным, если он обратим и $A^{-1} \in\left\{\mathscr{G}_{1}\right\}$. Множество строго положительных операторов в $\mathscr{G}_{1}$ обозначим символом $\left\{\mathscr{G}_{1}\right\}_{>}$. Пусть операторы $A, B \in\left\{\mathscr{G}_{1}\right\}_{H}$. Неравенство $A \geqslant B$ (соответственно $A>B$ ) означает, что $A-B \in\left\{\mathscr{G}_{1}\right\}_{\geqslant}$(соответственно $A-B \in\left\{\mathscr{G}_{1}\right\}_{>}$).

Тож дественный и нулевой операторы, действующие в некотором гильбертовом пространстве $\mathscr{G}_{1}$, обозначим символами $I \mathscr{G}_{1}$ и $0 \mathscr{G}_{1}$. Нулевой оператор, действующий из гильбертова пространства $\mathscr{G}_{1}$ в гильбертово пространство $\mathscr{G}_{2}$, обозначим символом $0 \mathscr{G}_{1} \mathscr{G}_{2}$.

Пусть заданы операторы $K_{1} \in\left\{\mathscr{G}_{1}\right\} \geqslant, K_{2} \in\left\{\mathscr{G}_{2}\right\} \geqslant, L_{1}, L_{2} \in\left\{\mathscr{G}_{2}, \mathscr{G}_{1}\right\}, v_{1} \in$ $\left\{\mathscr{H}, \mathscr{G}_{1}\right\}, u_{2} \in\left\{\mathscr{H}, \mathscr{G}_{2}\right\}$. И пусть эти операторы удовлетворяют основному тождеству (OT)

$$
K_{1} L_{1}-L_{2} K_{2}=-v_{1} u_{2}^{*}
$$

ОПРЕДЕЛЕНИЕ 1. Упорядоченньй набор операторов

$$
\mathscr{P}=\left\{K_{1}, L_{1}, v_{1}, K_{2}, L_{2}, u_{2}\right\}
$$

удовлетворяющих ОТ (1), называется обобщенной интерполяционной задачей стилтьесовского типа, а пространства $\mathscr{G}_{1}, \mathscr{G}_{2}, \mathscr{H}$ называются масштабными пространствами.

Рассмотрим операторы

$$
T_{1}=L_{2} L_{1}^{*}, \quad T_{2}=L_{1}^{*} L_{2}, \quad u_{1}=L_{2} u_{2}, \quad v_{2}=L_{1}^{*} v_{1}
$$

Непосредственно из определений операторов $T_{1}$ и $T_{2}$ имеем

$$
T_{1} L_{2}=L_{2} T_{2}, \quad T_{2} L_{1}^{*}=L_{1}^{*} T_{1}
$$

Пусть операторы $T_{r}$ таковы, что о.ф. $R_{T_{r}}(z)=\left(I_{\mathscr{G}_{r}}-z T_{r}\right)^{-1}, r=1,2$, мероморфны в $\mathbb{C} \backslash \mathbb{R}_{+}$. Множество особых точек о.ф. $R_{T_{1}}$ и $R_{T_{2}}$ в $\mathbb{C} \backslash \mathbb{R}_{+}$обозначим символом $\mathscr{Z}$. И пусть $\overline{\mathscr{Z}}=\{z \in \mathbb{C}: \bar{z} \in \mathscr{Z}\}$. Из мероморфности $R_{T_{1}}$ и $R_{T_{2}}$ следует, что множества $\mathscr{Z}$ и $\overline{\mathscr{Z}}$ дискретны в $\mathbb{C} \backslash \mathbb{R}_{+}$, т.е. не имеют предельных точек в $\mathbb{C} \backslash \mathbb{R}_{+}$. 
Из этих определений и из (4) следует, что

$$
\begin{gathered}
R_{T_{1}}(z) L_{2}=L_{2} R_{T_{2}}(z), \quad R_{T_{2}}(z) L_{1}^{*}=L_{1}^{*} R_{T_{1}}(z), \\
(t-z) R_{T_{r}}(z) T_{r} R_{T_{r}}(t)=R_{T_{r}}(t)-R_{T_{r}}(z) \quad \forall z, t \in \mathbb{C} \backslash\left\{\mathbb{R}_{+} \cup \mathscr{Z}\right\} .
\end{gathered}
$$

ОПРеДЕЛЕНИЕ 2. Оператор-функция $w: \mathbb{C}_{+} \rightarrow\{\mathscr{H}\}$ называется неванлинновской, если она голоморфна в $\mathbb{C}_{+}$и $\left\{w(z)-w^{*}(z)\right\} /(2 i) \geqslant 0 \mathscr{H} \forall z \in \mathbb{C}_{+}$.

Класс всех таких о.ф. обозначим $\mathscr{R}$.

ОПРЕДЕЛЕниЕ 3 . Неванлинновская о.ф. $s: \mathbb{C}_{+} \rightarrow\{\mathscr{H}\}$ назьвается стилтьесовской, если она определена и непрерывна на полуоси $\mathbb{R}_{-}$и $s(x) \geqslant 0 \mathscr{H} \forall x \in \mathbb{R}_{-}$.

Каждая стилтьесовская о.ф. $s$ по принципу симметрии $s(z)=s^{*}(\bar{z})$ продолжается до голоморфной в $\mathbb{C} \backslash \mathbb{R}_{+}$о.ф. Класс всех продолженных по принципу симметрии стилтьесовских о.ф. обозначим символом $\mathscr{S}$.

В [16] доказано, что стилтьесовские о.ф. можно описать с помощью специальных интегральных представлений.

Лемма 1. Для любой о.ф. $s \in \mathscr{S}$ найдется оператор $\gamma \in\{\mathscr{H}\} \geqslant$ и монотонно возрастающая о.ф. $\sigma: \mathbb{R}_{+} \rightarrow\{\mathscr{H}\}_{H}$ такие, что существует интеграл $\int_{0}^{\infty} \frac{d \sigma(t)}{t+1} u$

$$
s(z)=\gamma+\int_{0}^{\infty} \frac{d \sigma(t)}{t-z} \quad \forall z \in \mathbb{C} \backslash \mathbb{R}_{+} .
$$

Это представление единственно с точностью до нормировки о.ф. $\sigma$ в точках ее разрывов.

ОпРЕДЕЛЕниЕ 4. Оператор-функция $s \in \mathscr{S}$ называется решением обобщенной интерполяиионной задачи (2), если она удовлетворяет следуюшей системе основных матричных неравенств (ОМН) В.П. Потапова:

$$
\left[\begin{array}{c|c}
K_{r} & R_{T_{r}}(z)\left\{v_{r} z^{r-1} s(z)-u_{r}\right\} \\
\hline * & \left\{z^{r-1} s(z)-\bar{z}^{r-1} s^{*}(z)\right\} /\{z-\bar{z}\}
\end{array}\right] \geqslant 0 \mathscr{G}_{r} \oplus \mathscr{H}, \quad z \in \mathbb{C}_{ \pm} \backslash \mathscr{Z}, \quad r=1,2 .
$$

Множество всех решений обобщенной интерполяционной задачи (2) обозначим символом $\mathscr{F}$.

ОПРЕДЕЛЕНИЕ 5. Обобщенная интерполяционная задача (2) называется вполне неопределенной, если

$$
\begin{gathered}
K_{1} \in\left\{\mathscr{G}_{1}\right\}_{>}, \quad K_{2} \in\left\{\mathscr{G}_{2}\right\}_{>} \quad \forall h \in \mathscr{H}, \quad v_{r} h=0 \in \mathscr{G}_{r} \Leftrightarrow h=0 \in \mathscr{H} \\
\forall h \in \mathscr{H}, \quad u_{r} h=0 \in \mathscr{G}_{r} \Leftrightarrow h=0 \in \mathscr{H}, \quad r=1,2 .
\end{gathered}
$$

Далее в этой статье мы будем рассматривать только вполне неопределенную обобщенную интерполяционную задачу (2). 
Рассмотрим две о.ф.

$$
\begin{aligned}
& U_{1}(z)=\left[\begin{array}{cc}
\alpha_{1} & \beta_{1} \\
\gamma_{1} & \delta_{1}
\end{array}\right]=\left[\begin{array}{c|c}
I_{\mathscr{H}}+z v_{2}^{*} R_{T_{2}^{*}}(z) K_{2}^{-1} u_{2} & -z v_{1}^{*} R_{T_{1}^{*}}(z) K_{1}^{-1} v_{1} \\
\hline u_{2}^{*} R_{T_{2}^{*}}(z) K_{2}^{-1} u_{2} & I_{\mathscr{H}}-z u_{1}^{*} R_{T_{1}^{*}}(z) K_{1}^{-1} v_{1}
\end{array}\right] \\
& U_{2}(z)=\left[\begin{array}{c|c}
I_{\mathscr{H}}+z v_{2}^{*} R_{T_{2}^{*}}(z) K_{2}^{-1} u_{2} & -v_{1}^{*} R_{T_{1}^{*}}(z) K_{1}^{-1} v_{1} \\
\hline z u_{2}^{*} R_{T_{2}^{*}}(z) K_{2}^{-1} u_{2} & I_{\mathscr{H}}-z u_{1}^{*} R_{T_{1}^{*}}(z) K_{1}^{-1} v_{1}
\end{array}\right]
\end{aligned}
$$

Здесь $R_{T_{r}^{*}}(z)=\left(I_{\mathscr{G}_{r}}-z T_{r}^{*}\right)^{-1}, r=1,2$, а разбиение на блоки понимаем в соответствии с представлением $\mathscr{H} \oplus \mathscr{H}$. Ясно, что о.ф. $U_{1}$ и $U_{2}$ голоморфны в $\mathbb{C} \backslash\left\{\mathbb{R}_{+} \cup \overline{\mathscr{Z}}\right\}$ и $U_{1}, U_{2}: \mathbb{C} \backslash\left\{\mathbb{R}_{+} \cup \overline{\mathscr{Z}}\right\} \rightarrow\{\mathscr{H} \oplus \mathscr{H}\}$.

Оператор-функция $U_{1}$ называется резольвентной матрицей обобщенной интерполяционной задачи (2).

Введем операторы в пространстве $\mathscr{H} \oplus \mathscr{H}$ :

$$
J=\left[\begin{array}{cc}
0_{\mathscr{H}} & -i I_{\mathscr{H}} \\
i I_{\mathscr{H}} & 0_{\mathscr{H}}
\end{array}\right], \quad J_{\pi}=\left[\begin{array}{cc}
0_{\mathscr{H}} & I_{\mathscr{H}} \\
I_{\mathscr{H}} & 0_{\mathscr{H}}
\end{array}\right]
$$

Непосредственные вычисления с использованием (1), (3)-(5) приводят к следующим выражениям для $J$-форм о.ф. $U_{r}, r=1,2$,

$$
J-U_{r}(z) J U_{r}^{*}(\lambda)=i(z-\bar{\lambda})\left[\begin{array}{c}
v_{r}^{*} \\
u_{r}^{*}
\end{array}\right] R_{T_{r}^{*}}(z) K_{r}^{-1} R_{T_{r}^{*}}^{*}(\lambda)\left[v_{r}, u_{r}\right] .
$$

Умножим последнее равенство справа на $J$ и подставим в него $\bar{z}$ вместо $\lambda$. Учитьвая равенство $J^{2}=I_{\mathscr{H}} \oplus \mathscr{H}$, приходим к принципу симметрии

$$
U_{r}^{-1}(z)=J U_{r}^{*}(\bar{z}) J, \quad r=1,2 .
$$

Подставим в (10) $\bar{z}$ вместо $z$ и $\lambda$ и затем умножим (10) слева и справа на $J$. Из принципа симметрии и очевидного равенства $R_{T_{r}^{*}}^{*}(\bar{z})=R_{T_{r}}(z)$ следует, что

$$
J-U_{r}^{-1^{*}}(z) J U_{r}^{-1}(z)=i(\bar{z}-z) J\left[\begin{array}{c}
v_{r}^{*} \\
u_{r}^{*}
\end{array}\right] R_{T_{r}}^{*}(z) K_{r}^{-1} R_{T_{r}}(z)\left[v_{r}, u_{r}\right] J
$$

Непосредственные вычисления с использованием (1), (3)-(5) приводят к следующему выражению для $J_{\pi}$-формы о.ф. $U_{1}$ :

$$
\begin{aligned}
U_{1}(z) & J_{\pi} U_{1}^{*}(z)-J_{\pi} \\
= & {\left[\begin{array}{cc}
\alpha(z) \beta^{*}(z)+\beta(z) \alpha^{*}(z) & -I_{\mathscr{H}}+\alpha(z) \delta^{*}(z)+\beta(z) \gamma^{*}(z) \\
-I_{\mathscr{H}}+\gamma(z) \beta^{*}(z)+\delta(z) \alpha^{*}(z) & \gamma(z) \delta^{*}(z)+\delta(z) \gamma^{*}(z)
\end{array}\right] } \\
= & -(z+\bar{z})\left[\begin{array}{c}
v_{1}^{*} \\
u_{1}^{*}
\end{array}\right] R_{T_{1}^{*}(z) K_{1}^{-1} R_{T_{1}^{*}}^{*}(z)\left[v_{1}, u_{1}\right]} \\
& +2\left[\begin{array}{c}
z v_{2}^{*} \\
u_{2}^{*}
\end{array}\right] R_{T_{2}^{*}}(z) K_{2}^{-1} R_{T_{2}^{*}}^{*}(z)\left[\bar{z} v_{2}, u_{2}\right] .
\end{aligned}
$$


ОПРЕДЕЛЕНИЕ 6 . Пусть о.ф. $p(z), q(z)$ мероморфны в $\mathbb{C} \backslash \mathbb{R}_{+}$и принимают значения в $\{\mathscr{H}\}$. Пара $\operatorname{col}[p(z) q(z)]$ называется стилтьесовской, если для нее существует дискретное в $\mathbb{C} \backslash \mathbb{R}_{+}$множество точек $\mathscr{D}_{p q}$ такое, что

1) $p^{*}(z) p(z)+q^{*}(z) q(z)>0 \mathscr{H} \forall z \in \mathbb{C} \backslash\left\{\mathbb{R}_{+} \cup \mathscr{D}_{p q}\right\}$;

2) $\left[p^{*}(z), q^{*}(z)\right] \frac{J}{i(\bar{z}-z)}\left[\begin{array}{c}p(z) \\ q(z)\end{array}\right] \geqslant 0_{\mathscr{H}} \forall z \in \mathbb{C}_{ \pm} \backslash \mathscr{D}_{p q}$;

3) $\left[p^{*}(z), \bar{z} q^{*}(z)\right] \frac{J}{i(\bar{z}-z)}\left[\begin{array}{c}p(z) \\ z q(z)\end{array}\right] \geqslant 0 \mathscr{H} \forall z \in \mathbb{C}_{ \pm} \backslash \mathscr{D}_{p q}$.

Можно доказать, что в этом определении условие 3) можно заменить эквивалентньп условием

$\left.3^{\prime}\right)\left[p^{*}(z), q^{*}(z)\right] J_{\pi}\left[\begin{array}{l}p(z) \\ q(z)\end{array}\right] \geqslant 0_{\mathscr{H}} \forall z \in \Pi_{-} \backslash \mathscr{D}_{p q}$.

Легко видеть, что всякая стилтьесовская о.ф. $s \in \mathscr{S}$ порождает стилтьесовскую пару $\operatorname{col}\left[\begin{array}{ll}I \mathscr{H} & s(z)\end{array}\right]$. Однако не всякая стилтьесовская пара представляется в таком виде. Например, пара $\operatorname{col}\left[0_{\mathscr{H}} I_{\mathscr{H}}\right]$ является стилтьесовской, но она не порождается стилтьесовской о.ф.

На множестве стилтьесовских пар введем отношение эквивалентности: пары $\operatorname{col}\left[p_{1}(z) q_{1}(z)\right]$ и $\operatorname{col}\left[p_{2}(z) \quad q_{2}(z)\right]$ назьваются эквивалентными, если существует о.ф. $Q(z)$, принимающая значения в $\{\mathscr{H}\}$, такая, что в $\mathbb{C} \backslash \mathbb{R}_{+}$мероморфны обе о.ф. $Q(z),(Q(z))^{-1}$ и $p_{1}(z)=p_{2}(z) Q(z), q_{1}(z)=q_{2}(z) Q(z)$. Множество классов эквивалентности стилтьесовских пар обозначим через $\mathscr{S}_{\infty}$.

В [7] доказано, что система ОМН (7) эквивалентна следующей факторизованной системе ОМН В.П. Потапова $(r=1,2)$ :

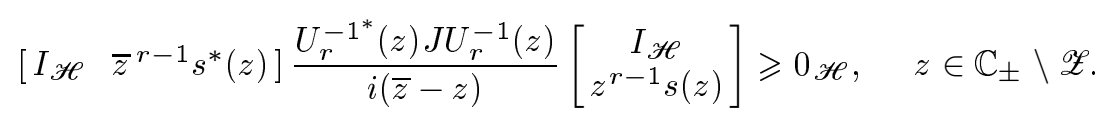

Множество $\mathscr{F}$ всех решений обобщенной интерполяционной задачи (2) можно описать и в терминах дробно-линейных преобразований (см. [17], [18]). А именно формула

$$
s(z)=\left\{\gamma_{1}(z) p(z)+\delta_{1}(z) q(z)\right\} \cdot\left\{\alpha_{1}(z) p(z)+\beta_{1}(z) q(z)\right\}^{-1}
$$

устанавливает биективное соответствие между $\mathscr{F}$ и $\mathscr{S}_{\infty}$.

\section{§2. Главные решения}

Легко видеть, что каждая из пар col $\left[\begin{array}{ll}I_{\mathscr{H}} & 0 \mathscr{H}\end{array}\right]$ и col $\left[\begin{array}{ll}0 \mathscr{H} & I_{\mathscr{H}}\end{array}\right]$ является стилтьесовской парой. Подставляя эти пары в дробно-линейное преобразование (14), получим два главных решения обобшенной интерполяционной задачи (2):

$$
s_{F}(z)=\gamma_{1}(z) \alpha_{1}^{-1}(z) \in \mathscr{F}, \quad s_{K}(z)=\delta_{1}(z) \beta_{1}^{-1}(z) \in \mathscr{F} .
$$

При этом о.ф. $s_{F}$ называется решением Фридрихса, а о.ф. $s_{K}-$ решением Крейна обобщенной интерполяционной задачи. Для случая скалярной проблемы моментов такие термины были предложены в статье [19]. Мы вводим и изучаем решения Фридрихса и Крейна для обобшенной интерполяционной задачи (2). 
Теорема 1. Решения Фридрихса и Крейна обобщенной вполне неопределенной интерполяционной задачи (2) допускают представления

$$
s_{F}(z)=u_{2}^{*}\left(K_{2}-z L_{1}^{*} K_{1} L_{1}\right)^{-1} u_{2}, \quad s_{K}(z)=\left\{v_{1}^{*}\left(L_{2} K_{2} L_{2}^{*}-z K_{1}\right)^{-1} v_{1}\right\}^{-1} .
$$

ДокАЗАТЕЛьство. Из равенства

$$
s_{F}(z)=-z^{-1} u_{2}^{*} K_{2}^{-1 / 2}\left(K_{2}^{-1 / 2} L_{1}^{*} K_{1} L_{1} K_{2}^{-1 / 2}-z^{-1} I \mathscr{G}_{2}\right)^{-1} K_{2}^{-1 / 2} u_{2}
$$

и свойств резольвенты неотрицательного оператора следует, что о.ф. $s_{F}(z)$ из (16) определена и голоморфна в $\mathbb{C} \backslash \mathbb{R}_{+}$. Далее, имеем

$$
\begin{aligned}
u_{2}^{*} & \left(K_{2}-z L_{1}^{*} K_{1} L_{1}\right)^{-1} u_{2} \cdot \alpha_{1}(z) \\
& =u_{2}^{*}\left(K_{2}-z L_{1}^{*} K_{1} L_{1}\right)^{-1} u_{2}\left\{I_{\mathscr{H}}+z v_{2}^{*} R_{T_{2}^{*}}(z) K_{2}^{-1} u_{2}\right\} \\
& =u_{2}^{*}\left(K_{2}-z L_{1}^{*} K_{1} L_{1}\right)^{-1}\left\{K_{2}\left(I \mathscr{G}_{2}-z T_{2}^{*}\right)+z u_{2} v_{2}^{*}\right\} R_{T_{2}^{*}}(z) K_{2}^{-1} u_{2} \\
& =u_{2}^{*}\left(K_{2}-z L_{1}^{*} K_{1} L_{1}\right)^{-1}\left\{K_{2}\left(I \mathscr{G}_{2}-z T_{2}^{*}\right)+z u_{2} v_{1}^{*} L_{1}\right\} R_{T_{2}^{*}}(z) K_{2}^{-1} u_{2} \\
& =u_{2}^{*}\left(K_{2}-z L_{1}^{*} K_{1} L_{1}\right)^{-1}\left\{K_{2}\left(I \mathscr{G}_{2}-z T_{2}^{*}\right)+z\left(K_{2} L_{2}^{*}-L_{1}^{*} K_{1}\right) L_{1}\right\} R_{T_{2}^{*}}(z) K_{2}^{-1} u_{2} \\
& =u_{2}^{*}\left(K_{2}-z L_{1}^{*} K_{1} L_{1}\right)^{-1}\left\{K_{2}-z L_{1}^{*} K_{1} L_{1}\right\} R_{T_{2}^{*}}(z) K_{2}^{-1} u_{2} \\
& =u_{2}^{*} R_{T_{2}^{*}}(z) K_{2}^{-1} u_{2}=\gamma_{1}(z) .
\end{aligned}
$$

В этой цепочке равенств первое вытекает из (9), третье - из (3), четвертое - из (1), пятое - из (3), седьмое - из (9). Итак,

$$
s_{F}(z)=\gamma_{1}(z) \alpha_{1}^{-1}(z)=u_{2}^{*}\left\{K_{2}-z L_{1}^{*} K_{1} L_{1}\right\}^{-1} u_{2}
$$

Первое из равенств (16) доказано. Второе из равенств (16) доказывается аналогичным образом.

ТЕОРЕМА 2. Пусть дана обобщенная вполне неопределенная интерполяиионная задача (2) и Я્F обозначает множество ее решений. И пусть, далее, $s_{F}$ $u s_{K}-$ главные решения задачи (2). Тогда о.ф. $\left\{s_{K}(z)-s_{F}(z)\right\}^{-1}$ определена и голоморфна при $z \in \mathbb{C} \backslash\left\{\mathbb{R}_{+} \cup \mathscr{Z} \cup \overline{\mathscr{Z}}\right\} u$

$$
\begin{array}{ll}
\text { 1) } \quad & \left\{s_{K}(z)-s_{F}(z)\right\}^{-1} \\
& =-z v_{1}^{*} R_{T_{1}^{*}}(z) K_{1}^{-1} R_{T_{1}}(z) v_{1}+z^{2} v_{2}^{*} R_{T_{2}^{*}}(z) K_{2}^{-1} R_{T_{2}}(z) v_{2} ; \\
& s_{K}(x)>s_{F}(x) \quad \forall x \in \mathbb{R}_{-} \backslash \mathscr{Z}, \quad s_{K}(x) \geqslant s_{F}(x) \quad \forall x \in \mathbb{R}_{-} ; \\
\text {3) } \quad 0_{\mathscr{H}}<s_{F}(x) \leqslant s(x) \leqslant s_{K}(x) \quad \forall s \in \mathscr{F}, \forall x \in \mathbb{R}_{-} .
\end{array}
$$

ДокАЗАТЕльство. 1) Зафиксируем произвольную точку $x \in \mathbb{R}_{-} \backslash \mathscr{Z}$. Подставим это значение $x$ в (10) вместо $z$ и $\lambda$. Получим $J-U_{1}(x) J U_{1}^{*}(x)=0 \mathscr{H} \oplus \mathscr{H}$. Но тогда (см. [15]) и $J-U_{1}^{*}(x) J U_{1}(x)=0 \mathscr{H} \oplus \mathscr{H}$. Отсюда и из блочного представления (9) следует, что

$$
\left[\begin{array}{cc}
\alpha_{1}^{*}(x) \gamma_{1}(x)-\gamma_{1}^{*}(x) \alpha_{1}(x) & \alpha_{1}^{*}(x) \delta_{1}(x)-\gamma_{1}^{*}(x) \beta_{1}(x) \\
\beta_{1}^{*}(x) \gamma_{1}(x)-\delta_{1}^{*}(x) \alpha_{1}(x) & \beta_{1}^{*}(x) \delta_{1}(x)-\delta_{1}^{*}(x) \beta_{1}(x)
\end{array}\right]=\left[\begin{array}{cc}
0 \mathscr{H} & I_{\mathscr{H}} \\
-I_{\mathscr{H}} & 0_{\mathscr{H}}
\end{array}\right]
$$


Таким образом, $-\beta_{1}^{*}(x) \gamma_{1}(x)+\delta_{1}^{*}(x) \alpha_{1}(x)=I_{\mathscr{H}}$. В силу $(6) s_{K}(x)=s_{K}^{*}(x)$. Поэтому

$$
\begin{aligned}
s_{K}(x) & -s_{F}(x)=\beta_{1}^{-1^{*}}(x) \delta_{1}^{*}(x)-\gamma_{1}(x) \alpha_{1}^{-1}(x) \\
& =\beta_{1}^{-1^{*}}(x)\left\{\delta_{1}^{*}(x) \alpha_{1}(x)-\beta_{1}^{*}(x) \gamma_{1}(x)\right\} \alpha_{1}^{-1}(x)=\beta_{1}^{-1^{*}}(x) \alpha_{1}^{-1}(x) .
\end{aligned}
$$

Следовательно,

$$
\begin{aligned}
\left\{s_{K}(x)-\right. & \left.s_{F}(x)\right\}^{-1}=\alpha_{1}(x) \beta_{1}^{*}(x) \\
= & -x\left\{I_{\mathscr{H}}+x v_{2}^{*} R_{T_{2}^{*}}(x) K_{2}^{-1} u_{2}\right\}\left\{v_{1}^{*} K_{1}^{-1} R_{T_{1}}(x) v_{1}\right\} \\
= & -x\left\{v_{1}^{*} K_{1}^{-1} R_{T_{1}}(x) v_{1}+x v_{2}^{*} R_{T_{2}^{*}}(x) K_{2}^{-1}\left(-L_{1}^{*} K_{1}+K_{2} L_{2}^{*}\right) K_{1}^{-1} R_{T_{1}}(x) v_{1}\right\} \\
= & -x\left\{v_{1}^{*} K_{1}^{-1} R_{T_{1}}(x) v_{1}-x v_{2}^{*} R_{T_{2}^{*}}(x) K_{2}^{-1} L_{1}^{*} R_{T_{1}}(x) v_{1}\right. \\
& \left.+x v_{2}^{*} R_{T_{2}^{*}}(x) L_{2}^{*} K_{1}^{-1} R_{T_{1}}(x) v_{1}\right\} \\
=- & x\left\{v_{1}^{*} K_{1}^{-1} R_{T_{1}}(x) v_{1}-x v_{2}^{*} R_{T_{2}^{*}}(x) K_{2}^{-1} L_{1}^{*} R_{T_{1}}(x) v_{1}\right. \\
& \left.+x v_{1}^{*} L_{1} L_{2}^{*} R_{T_{1}^{*}}(x) K_{1}^{-1} R_{T_{1}}(x) v_{1}\right\} \\
=- & x\left\{v_{1}^{*} K_{1}^{-1} R_{T_{1}}(x) v_{1}-x v_{2}^{*} R_{T_{2}^{*}}(x) K_{2}^{-1} L_{1}^{*} R_{T_{1}}(x) v_{1}\right. \\
& \left.+x v_{1}^{*} T_{1}^{*} R_{T_{1}^{*}}(x) K_{1}^{-1} R_{T_{1}}(x) v_{1}\right\} \\
=- & x\left\{v_{1}^{*}\left[I_{G_{1}}-x T_{1}^{*}+x T_{1}^{*}\right] R_{T_{1}^{*}}(x) K_{1}^{-1} R_{T_{1}}(x) v_{1}\right. \\
& \left.-x v_{2}^{*} R_{T_{2}^{*}}(x) K_{2}^{-1} L_{1}^{*} R_{T_{1}}(x) v_{1}\right\} \\
= & -x\left\{v_{1}^{*} R_{T_{1}^{*}}(x) K_{1}^{-1} R_{T_{1}}(x) v_{1}-x v_{2}^{*} R_{T_{2}^{*}}(x) K_{2}^{-1} L_{1}^{*} R_{T_{1}}(x) v_{1}\right\} \\
=- & x\left\{v_{1}^{*} R_{T_{1}^{*}}(x) K_{1}^{-1} R_{T_{1}}(x) v_{1}-x v_{2}^{*} R_{T_{2}^{*}}(x) K_{2}^{-1} R_{T_{2}}(x) v_{2}\right\} .
\end{aligned}
$$

В этой цепочке равенств второе равенство следует из (9), третье - из (1), пятое, шестое и девятое - из (3), (4) и (5). Таким образом, при $x \in \mathbb{R}_{-} \backslash \mathscr{Z}$

$$
\left\{s_{K}(x)-s_{F}(x)\right\}^{-1}=-x\left\{v_{1}^{*} R_{T_{1}^{*}}(x) K_{1}^{-1} R_{T_{1}}(x) v_{1}-x v_{2}^{*} R_{T_{2}^{*}}(x) K_{2}^{-1} R_{T_{2}}(x) v_{2}\right\} .
$$

Отсюда в силу аналитичности следует (17).

2) Пусть $x \in \mathbb{R}_{-} \backslash \mathscr{Z}$. Имеем

$$
\begin{aligned}
& \left\{s_{K}(x)-s_{F}(x)\right\}^{-1} \\
& \quad=-x\left\{v_{1}^{*} R_{T_{1}^{*}}(x) K_{1}^{-1} R_{T_{1}}(x) v_{1}-x v_{2}^{*} R_{T_{2}^{*}}(x) K_{2}^{-1} R_{T_{2}}(x) v_{2}\right\}>0 \mathscr{H} .
\end{aligned}
$$

Здесь мы последовательно воспользовались $(17),(8)$ и тем фактом, что при $x \in$ $\mathbb{R}_{-} \backslash \mathscr{Z}$ о.ф.. $R_{T_{1}}(x)$ и $R_{T_{2}}(x)$ являются обратимыми операторами. Таким образом, доказано первое из неравенств (18). Второе из неравенств (18) получается продолжением по непрерывности первого из неравенств (18) в точки $x \in \mathbb{R}_{-} \cap \mathscr{Z}$.

$3)$ Пусть о.ф. $s \in \mathscr{F}$. Тогда она допускает представление (14) с некоторой стилтьесовской парой $\operatorname{col}\left[\begin{array}{ll}p & q\end{array}\right]$. Пусть $x \in \mathbb{R}_{-} \backslash\left\{\mathscr{D}_{p q} \cup \mathscr{Z}\right\}$. Имеем

$$
\begin{gathered}
s_{K}(x)-s(x)=\delta_{1}(x) \beta_{1}^{-1}(x)-\left\{\gamma_{1}(x) p(x)+\delta_{1}(x) q(x)\right\}\left\{\alpha_{1}(x) p(x)+\beta_{1}(x) q(x)\right\}^{-1} \\
=\beta_{1}^{-1^{*}}(x) \delta_{1}^{*}(x)-\left\{\gamma_{1}(x) p(x)+\delta_{1}(x) q(x)\right\}\left\{\alpha_{1}(x) p(x)+\beta_{1}(x) q(x)\right\}^{-1}
\end{gathered}
$$




$$
\begin{aligned}
= & \beta_{1}^{-1^{*}}(x)\left\{\left[\delta_{1}^{*}(x) \alpha_{1}(x)-\beta_{1}^{*}(x) \gamma_{1}(x)\right] p(x)+\left[\delta_{1}^{*}(x) \beta_{1}(x)-\beta_{1}^{*}(x) \delta_{1}(x)\right] q(x)\right\} \\
& \times\left\{\alpha_{1}(x) p(x)+\beta_{1}(x) q(x)\right\}^{-1} \\
= & \beta_{1}^{-1^{*}}(x) p(x)\left\{\alpha_{1}(x) p(x)+\beta_{1}(x) q(x)\right\}^{-1} .
\end{aligned}
$$

В этой цепочке равенств второе равенство следует из эрмитовости $s_{K}(x)$, а последнее - из (20). Окончательно

$$
s_{K}(x)-s(x)=\beta_{1}^{-1^{*}}(x) p(x)\left\{\alpha_{1}(x) p(x)+\beta_{1}(x) q(x)\right\}^{-1} .
$$

Таким образом, разность $s_{K}(x)-s(x)$ представлена в виде дробно-линейного преобразования над стилтьесовской парой $\operatorname{col}[p(z) q(z))]$. Матрица дробно-линейного преобразования в $(22)$ имеет вид

$$
\widetilde{U}(x)=\left[\begin{array}{cc}
\alpha_{1}(x) & \beta_{1}(x) \\
\beta_{1}^{-1^{*}}(x) & 0 \mathscr{H}
\end{array}\right] .
$$

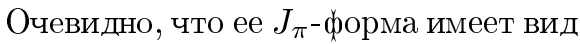

$$
\widetilde{U}^{*}(x) J_{\pi} \widetilde{U}(x)-J_{\pi}=\left[\begin{array}{cc}
\beta_{1}^{-1}(x)\left[\beta_{1}(x) \alpha_{1}^{*}(x)+\alpha_{1}(x) \beta^{*}(x)\right] \beta_{1}^{-1^{*}}(x) & 0 \mathscr{H} \\
0 \mathscr{H} & 0 \mathscr{H}
\end{array}\right] .
$$

Из (12) следует, что $U_{1}(x) J_{\pi} U_{1}^{*}(x)-J_{\pi} \geqslant 0 \mathscr{H} \oplus \mathscr{H}$. Но тогда, снова из (12) имеем $\beta_{1}(x) \alpha_{1}^{*}(x)+\alpha_{1}(x) \beta_{1}^{*}(x) \geqslant 0 \mathscr{H}$. Поэтому $\widetilde{U}^{*}(x) J_{\pi} \widetilde{U}(x) \geqslant J_{\pi}$. С учетом этого неравенства получаем

$$
\begin{aligned}
& {\left[p^{*}(x) \alpha_{1}^{*}(x)+q^{*}(x) \beta_{1}^{*}(x), p^{*}(x) \beta_{1}^{-1}(x)\right] J_{\pi}\left[\begin{array}{c}
\alpha_{1}(x) p(x)+\beta_{1}(x) q(x) \\
\beta_{1}^{-1^{*}}(x) p(x)
\end{array}\right]} \\
& =\left[p^{*}(x), q^{*}(x)\right]\left[\begin{array}{cc}
\alpha_{1}^{*}(x) & \beta_{1}^{-1}(x) \\
\beta_{1}^{*}(x) & 0 \mathscr{H}
\end{array}\right] J_{\pi}\left[\begin{array}{cc}
\alpha_{1}(x) & \beta_{1}(x) \\
\beta_{1}^{-1^{*}}(x) & 0 \mathscr{H}
\end{array}\right]\left[\begin{array}{c}
p(x) \\
q(x)
\end{array}\right] \\
& =\left[p^{*}(x), q^{*}(x)\right] \widetilde{U}^{*}(x) J_{\pi} \widetilde{U}(x)\left[\begin{array}{l}
p(x) \\
q(x)
\end{array}\right] \geqslant\left[p^{*}(x), q^{*}(x)\right] J_{\pi}\left[\begin{array}{l}
p(x) \\
q(x)
\end{array}\right] \geqslant 0 \mathscr{H} .
\end{aligned}
$$

Последнее неравенство вытекает из определения 6. Таким образом,

$$
\left[p^{*}(x) \alpha_{1}^{*}(x)+q^{*}(x) \beta_{1}^{*}(x), p^{*}(x) \beta_{1}^{-1}(x)\right] J_{\pi}\left[\begin{array}{c}
\alpha_{1}(x) p(x)+\beta_{1}(x) q(x) \\
\beta_{1}^{-1^{*}}(x) p(x)
\end{array}\right] \geqslant 0 \mathscr{H} .
$$

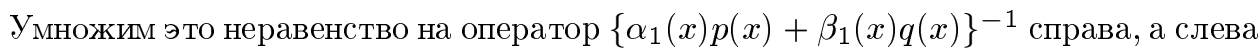
- на сопряженный оператор. С учетом (22) получим

$$
\left[I_{\mathscr{H}}, s_{K}^{*}(x)-s^{*}(x)\right] J_{\pi}\left[\begin{array}{c}
I_{\mathscr{H}} \\
s_{K}(x)-s(x)
\end{array}\right] \geqslant 0 \mathscr{H}
$$

Или, учитывая вид $J_{\pi}$,

$$
s_{K}^{*}(x)-s^{*}(x)+s_{K}(x)-s(x) \geqslant 0 \mathscr{H} .
$$


Оператор-функции $s_{K}$ и $s$ принадлежат $\mathscr{F} \subset \mathscr{S}$. Из определения класса $\mathscr{S}$ следует, что $s_{K}(x)$ и $s(x)$ эрмитовы. Но тогда из последнего неравенства имеем $s_{K}(x) \geqslant$ $s(x)$. Это неравенство продолжается в точки $x \in \mathbb{R}_{-} \cap\left(\mathscr{D}_{p q} \cup \mathscr{Z}\right)$ по непрерывности. Неравенство $s_{F}(x) \leqslant s(x)$ доказывается аналогичным образом. Неравенство $0 \mathscr{H}<s_{F}(x) \forall x \in \mathbb{R}_{-}$следует из (16) и (8).

Пусть $A, B \in\{\mathscr{H}\}_{>}$и выполнено условие $A \leqslant B$. Операторньм интервалом называется

$$
[A, B]=\left\{\kappa \in\{\mathscr{H}\}_{>}: A \leqslant \kappa \leqslant B\right\} .
$$

Число $\operatorname{rank}\{B-A\}$ называется рангом операторного интервала (под рангом оператора мы понимаем ранг его матрицы в любом базисе пространства $\mathscr{H}$ ).

Интервал $[A, B]$ называется невырожденным, если $A<B$.

ОпРЕДЕЛЕниЕ 7. Операторньй интервал

$$
\mathscr{I}(x)=\left[s_{F}(x), s_{K}(x)\right], \quad x \in \mathbb{R}_{-},
$$

называется интервалом Вейля в точке $x$.

Неравенство (19) означает, что $\{s(x): s \in \mathscr{F}\} \subset \mathscr{I}(x)$.

\section{§3. Упорядоченные интерполяционные задачи}

Пусть заданы две обобщенные интерполяционные задачи

$$
\mathscr{P}^{(1)}=\left\{K_{1}^{(1)}, L_{1}^{(1)}, v_{1}^{(1)}, K_{2}^{(1)}, L_{2}^{(1)}, u_{2}^{(1)}\right\}
$$

с масштабными пространствами $\left\{\mathscr{G}_{1}^{(1)}, \mathscr{G}_{2}^{(1)}, \mathscr{H}\right\}$ и

$$
\mathscr{P}^{(2)}=\left\{K_{1}^{(2)}, L_{1}^{(2)}, v_{1}^{(2)}, K_{2}^{(2)}, L_{2}^{(2)}, u_{2}^{(2)}\right\}
$$

с масштабными пространствами $\left\{\mathscr{G}_{1}^{(2)}, \mathscr{G}_{2}^{(2)}, \mathscr{H}\right\}$.

В обозначения объектов, относящихся к интерполяционной задаче $\mathscr{P}^{(1)}$ (соответственно $\mathscr{P}^{(2)}$ ), будем добавлять верхний индекс (1) (соответственно (2)).

ОПРЕДЕЛЕНИЕ 8. Обобщенные интерполяционные задачи (23) и (24) называются унитарно эквивалентныци, если сушествуют унитарные операторы $\mathscr{U}_{1}$ : $\mathscr{G}_{1}^{(1)} \rightarrow \mathscr{G}_{1}^{(2)}, \mathscr{U}_{2}: \mathscr{G}_{2}^{(1)} \rightarrow \mathscr{G}_{2}^{(2)}$ такие, что

$$
\begin{aligned}
& K_{1}^{(1)}=\mathscr{U}_{1}^{*} K_{1}^{(2)} \mathscr{U}_{1}, \quad L_{1}^{(1)}=\mathscr{U}_{1}^{*} L_{1}^{(2)} \mathscr{U}_{2}, \quad v_{1}^{(1)}=\mathscr{U}_{1}^{*} v_{1}^{(2)}, \\
& K_{2}^{(1)}=\mathscr{U}_{2}^{*} K_{2}^{(2)} \mathscr{U}_{2}, \quad L_{2}^{(1)}=\mathscr{U}_{1}^{*} L_{2}^{(2)} \mathscr{U}_{2}, \quad u_{2}^{(1)}=\mathscr{U}_{2}^{*} u_{2}^{(2)} \text {. }
\end{aligned}
$$

Отсюда и из формул (3) непосредственно вытекают соотношения

$$
\begin{aligned}
T_{1}^{(1)}= & \mathscr{U}_{1}^{*} T_{1}^{(2)} \mathscr{U}_{1}, \quad T_{2}^{(1)}=\mathscr{U}_{2}^{*} T_{2}^{(2)} \mathscr{U}_{2}, \quad u_{1}^{(1)}=\mathscr{U}_{1}^{*} u_{1}^{(2)}, \quad v_{2}^{(1)}=\mathscr{U}_{2}^{*} v_{2}^{(2)}, \\
& R_{T_{1}^{(1)^{*}}}(z)=\mathscr{U}_{1}^{*} R_{T_{1}^{(2) *}}(z) \mathscr{U}_{1}, \quad R_{T_{2}^{(1)^{*}}}(z)=\mathscr{U}_{2}^{*} R_{T_{2}^{(2) *}}(z) \mathscr{U}_{2} .
\end{aligned}
$$


ЛЕмма 2. Пусть задань две интерполяционные задачи $\mathscr{P}^{(r)}, r=1,2$. И пусть, далее, символь $U_{1}^{(r)}, U_{2}^{(r)}, \mathscr{F}^{(r)}, s_{F}^{(r)}, s_{K}^{(r)}$ обозначают соответствующие обтекты, связаннье с $r$-й интерполяционной задачей.

Если интерполяционнье задачи $\mathscr{P}^{(1)}$ и $\mathscr{P}^{(2)}$ унитарно әквивалентны, то

$$
\begin{aligned}
U_{1}^{(1)}(z) & =U_{1}^{(2)}(z), & U_{2}^{(1)}(z)=U_{2}^{(2)}(z), & \mathscr{F}(1)=\mathscr{F}^{(2)}, \\
s_{F}^{(1)}(z) & =s_{F}^{(2)}(z), & s_{K}^{(1)}(z)=s_{K}^{(2)}(z) & \forall z \in \mathbb{C} \backslash \mathbb{R}_{+} .
\end{aligned}
$$

ДокАЗАТЕЛЬСтво. Покажем, что совпадают резольвентные матрицы унитарно эквивалентных интерполяционных задач. Из (9) и (27) имеем

$$
\begin{aligned}
& U_{1}^{(1)}(z)=\left[\begin{array}{c|c}
I+z v_{2}^{(1)^{*}} R_{T_{2}^{(1) *}(z) K_{2}^{(1)^{-1}} u_{2}^{(1)}} & -z v_{1}^{(1)^{*}} R_{T_{1}^{(1)^{*}}(z) K_{1}^{(1)^{-1}} v_{1}^{(1)}} \\
\hline u_{2}^{(1)^{*}} R_{T_{2}^{(1)^{*}}}(z) K_{2}^{(1)^{-1}} u_{2}^{(1)} & I-z u_{1}^{(1)^{*}} R_{T_{1}^{(1)^{*}}}(z) K_{1}^{(1)^{-1}} v_{1}^{(1)}
\end{array}\right] \\
& =\left[\frac{I+z v_{2}^{(2)^{*}} \mathscr{U}_{2} \mathscr{U}_{2}^{*} R_{T_{2}^{(2) *}}(z) \mathscr{U}_{2} \mathscr{U}_{2}^{*} K_{2}^{(2)^{-1}} \mathscr{U}_{2} \mathscr{U}_{2}^{*} u_{2}^{(2)}}{u_{2}^{(2)^{*}} \mathscr{U}_{2} \mathscr{U}_{2}^{*} R_{T_{2}^{(2) *}}(z) \mathscr{U}_{2} \mathscr{U}_{2}^{*} K_{2}^{(2)^{-1}} \mathscr{U}_{2} \mathscr{U}_{2}^{*} u_{2}^{(2)}}\right.
\end{aligned}
$$

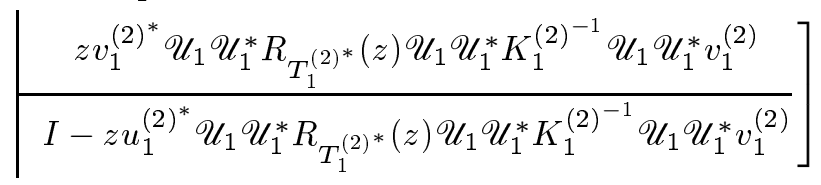

$$
\begin{aligned}
& =\left[\begin{array}{c|c}
I+z v_{2}^{(2)^{*}} R_{T_{2}^{(2) *}}(z) K_{2}^{(2)^{-1}} u_{2}^{(2)} & -z v_{1}^{(2)^{*}} R_{T_{1}^{(2) *}(z) K_{1}^{(2)^{-1}} v_{1}^{(2)}} \\
\hline u_{2}^{(2)^{*}} R_{T_{2}^{(2) *}}(z) K_{2}^{(2)^{-1}} u_{2}^{(2)} & I-z u_{1}^{(2)^{*}} R_{T_{1}^{(2) *}}(z) K_{1}^{(2)^{-1}} v_{1}^{(2)}
\end{array}\right] \\
& =U_{1}^{(2)}(z) \text {. }
\end{aligned}
$$

Итак, мы доказали, что $U_{1}^{(1)}(z)=U_{1}^{(2)}(z)$. Аналогичным образом доказываются равенства $U_{2}^{(1)}(z)=U_{2}^{(2)}(z)$. Отсюда следует, что для унитарно эквивалентных интерполяционных задач системы ОМН В.П. Потапова (13) совпадают. Поэтому $\mathscr{F}^{(1)}=\mathscr{F}^{(2)}$.

Главные решения интерполяционных задач $\mathscr{P}^{(1)}$ и $\mathscr{P}^{(2)}$ выражаются через элементы резольвентных матриц $U_{1}^{(1)}$ и $U_{1}^{(2)}$ по формулам (15). Но резольвентныематрицы $U_{1}^{(1)}$ и $U_{1}^{(2)}$ совпадают. Отсюда следуют две последние формулы в (28).

Пусть дана интерполяционная задача

$$
\mathscr{P}=\left\{K_{1}, L_{1}, v_{1}, K_{2}, L_{2}, u_{2}\right\},
$$

и пусть ее масштабные пространства $\mathscr{G}_{1}$ и $\mathscr{G}_{2}$ представлены в виде ортогональной суммы своих подпространств (подпространства $\widetilde{\mathscr{G}}_{r}$ предполагаются ненулевыми)

$$
\mathscr{G}_{r}=\widetilde{\mathscr{G}}_{r} \oplus \widehat{\mathscr{G}}_{r}, \quad r=1,2 .
$$


Это равенство понимается в смысле естественного изоморфизма $\mathscr{G}_{r}$ и $\widetilde{\mathscr{G}}_{r} \oplus \widehat{\mathscr{G}}_{r}$. А именно пусть $\widetilde{P}_{r}$ (соответственно $\widehat{P}_{r}$ ) обозначает ортопроектор пространства $\mathscr{G}_{r}$ на подпространство $\widetilde{\mathscr{G}}_{r}\left(\right.$ соответственно $\left.\widehat{\mathscr{G}}_{r}\right)$. Тогда соответствующий изоморфизм имеет вид

$$
\forall f \in \mathscr{G}_{r} \leftrightarrow \operatorname{col}\left[\widetilde{P}_{r} f, \widehat{P}_{r} f\right] \in \widetilde{\mathscr{G}}_{r} \oplus \widehat{\mathscr{G}}_{r} .
$$

Пусть выполнены условия

$$
L_{1} \widetilde{P}_{2}=\widetilde{P}_{1} L_{1} \widetilde{P}_{2}, \quad \widetilde{P}_{1} L_{2}=\widetilde{P}_{1} L_{2} \widetilde{P}_{2}
$$

В соответствии с (30)-(32) введем матричные обозначения $(r=1,2)$

$$
\begin{aligned}
& K_{r}=\left[\begin{array}{cc}
\widetilde{K}_{r} & B_{r} \\
B_{r}^{*} & C_{r}
\end{array}\right], \quad L_{1}=\left[\begin{array}{cc}
\widetilde{L}_{1} & D_{1} \\
0 \widetilde{\mathscr{G}}_{2} \widehat{\mathscr{G}}_{1} & \widehat{L}_{1}
\end{array}\right], \quad L_{2}=\left[\begin{array}{cc}
\widetilde{L}_{2} & 0_{\widehat{\mathscr{G}}_{2}} \widetilde{\mathscr{G}}_{1} \\
E_{2} & \widehat{L}_{2}
\end{array}\right], \\
& v_{1}=\left[\begin{array}{c}
\widetilde{v}_{1} \\
\check{v}_{1}
\end{array}\right], \quad u_{2}=\left[\begin{array}{c}
\widetilde{u}_{2} \\
\check{u}_{2}
\end{array}\right], \quad u_{1}=\left[\begin{array}{c}
\widetilde{L}_{2} \widetilde{u}_{2} \\
E_{2} \widetilde{u}_{2}+\widehat{L}_{2} \check{u}_{2}
\end{array}\right], \quad v_{2}=\left[\begin{array}{c}
\widetilde{L}_{1}^{*} \widetilde{v}_{1} \\
D_{1}^{*} \widetilde{v}_{1}+\widehat{L}_{1}^{*} \check{v}_{1}
\end{array}\right] \text {, } \\
& T_{1}=L_{2} L_{1}^{*}=\left[\begin{array}{cc}
\widetilde{L}_{2} \widetilde{L}_{1}^{*} & 0_{\widehat{\mathscr{G}}_{1}} \widetilde{\mathscr{G}}_{1} \\
E_{2} \widetilde{L}_{1}^{*}+\widehat{L}_{2} D_{1}^{*} & \widehat{L}_{2} \widehat{L}_{1}^{*}
\end{array}\right], \quad \widetilde{P}_{r}=\left[\begin{array}{cc}
I_{\widetilde{\mathscr{G}}_{r}} & 0_{\widehat{\mathscr{G}}_{r}} \widetilde{\mathscr{G}}_{r} \\
0_{\widetilde{G}_{r} \widehat{\mathscr{G}}_{r}} & 0_{\widehat{\mathscr{G}}_{r}}
\end{array}\right], \\
& T_{2}=L_{1}^{*} L_{2}=\left[\begin{array}{cc}
\widetilde{L}_{1}^{*} \widetilde{L}_{2} & 0_{\widehat{\mathscr{G}}_{2}} \widetilde{\mathscr{G}}_{2_{2}} \\
D_{1}^{*} \widetilde{L}_{2}+\widehat{L}_{1}^{*} E_{2}^{*} & \widehat{L}_{1}^{*} \widehat{L}_{2}
\end{array}\right], \quad \widehat{P}_{r}=\left[\begin{array}{cc}
0_{\widetilde{G}_{r}} & 0_{\widehat{\mathscr{G}}_{r} \widetilde{\mathscr{G}}_{r}} \\
0_{\widetilde{\mathscr{G}}_{r} \widehat{\mathscr{G}}_{r}} & I_{\widehat{\mathscr{G}}_{r}}
\end{array}\right] .
\end{aligned}
$$

Отметим, что операторы в левых частях двух первых равенств являются операторами в пространствах $\mathscr{G}_{r}$, а в правых частях записаны матричные представления тех же самых операторов в пространствах $\widetilde{\mathscr{G}}_{r} \oplus \widehat{\mathscr{G}}_{r}$. Матричные элементы операторов $K_{r}$ имеют вид

$$
\widetilde{K}_{r}=\left.\widetilde{P}_{r} K_{r}\right|_{\widetilde{\mathfrak{G}}_{r}}, \quad B_{r}=\left.\widetilde{P}_{r} K_{r}\right|_{\widehat{\mathfrak{G}}_{r}}, \quad B_{r}^{*}=\left.\widehat{P}_{r} K_{r}\right|_{\widetilde{\mathfrak{G}}_{r}}, \quad C_{r}=\left.\widehat{P}_{r} K_{r}\right|_{\widehat{\mathscr{G}}_{r}}
$$

В аналогичном смысле понимаем и остальные равенства в (33).

Из ОТ (1) и представлений (33) следует индуцированное ОТ

$$
\widetilde{K}_{1} \widetilde{L}_{1}-\widetilde{L}_{2} \widetilde{K}_{2}=-\widetilde{v}_{1} \widetilde{u}_{2}^{*}
$$

Непосредственно убеждаемся в том, что операторы

$$
\widetilde{\mathscr{P}}=\left\{\widetilde{K}_{1}, \widetilde{L}_{1}, \widetilde{v}_{1}, \widetilde{K}_{2}, \widetilde{L}_{2}, \widetilde{u}_{2}\right\}
$$

и масштабные пространства $\left\{\widetilde{\mathscr{G}}_{1}, \widetilde{\mathscr{G}}_{2}, \mathscr{H}\right\}$ создают вполне неопределенную интерполяционную задачу стилтьесовского типа.

ОПРЕДЕЛЕнИЕ 9. Интерполяционная задача (35) называется сужением интерполяционной задачи (29) на подпространства $\widetilde{\mathscr{G}}_{1}$ и $\widetilde{\mathscr{G}}_{2}$. 
ОПРЕДЕЛЕНИЕ 10. Пусть даны две обобщенные интерполяционные задачи стилтьесовского типа

$$
\begin{aligned}
& \mathscr{P}^{(1)}=\left\{K_{1}^{(1)}, L_{1}^{(1)}, v_{1}^{(1)}, K_{2}^{(1)}, L_{2}^{(1)}, u_{2}^{(1)}\right\}, \\
& \mathscr{P}^{(2)}=\left\{K_{1}^{(2)}, L_{1}^{(2)}, v_{1}^{(2)}, K_{2}^{(2)}, L_{2}^{(2)}, u_{2}^{(2)}\right\} .
\end{aligned}
$$

Говорят, что задача $\mathscr{P}^{(1)}$ содержится в задаче $\mathscr{P}^{(2)}$ или, что то же самое, задача $\mathscr{P}^{(2)}$ является продолжением задачи $\mathscr{P}^{(1)}$, если в масштабных пространствах $\mathscr{G}_{1}^{(2)}, \mathscr{G}_{2}^{(2)}$ сушествуют подпространства $\widetilde{\mathscr{G}}_{1}^{(2)}, \widetilde{\mathscr{G}}_{2}^{(2)}$, удовлетворяюшие условиям $(32)$, такие, что сужение задачи $\mathscr{P}(2)$ на подпространства $\mathscr{G}_{1}^{(2)}, \mathscr{G}_{2}^{(2)}$, обозначаемое символом $\widetilde{\mathscr{P}}^{(2)}$, унитарно эквивалентно задаче $\mathscr{P}^{(1)}$.

Коротко это записывается так: $\mathscr{P}^{(1)} \subset \mathscr{P}^{(2)}$.

В дальнейшем мы будем пользоваться хорошо известным свойством строго положительных операторов в гильбертовом пространстве. А именно пусть даны два гильбертовых пространства $\widetilde{\mathscr{G}}_{\text {и }} \widehat{\mathscr{G}}$. Тогда

$$
\left[\begin{array}{cc}
\widetilde{K} & B \\
B^{*} & C
\end{array}\right] \in\{\widetilde{\mathscr{G}} \oplus \widehat{\mathscr{G}}\}_{>} \Longrightarrow\left[\begin{array}{cc}
\widetilde{K} & B \\
B^{*} & C
\end{array}\right]^{-1} \geqslant\left[\begin{array}{cc}
\widetilde{K}^{-1} & 0_{\widehat{\mathscr{G}} \widetilde{\mathscr{G}}} \\
0_{\widetilde{G} \widehat{G}} & 0_{\widehat{\mathscr{G}}}
\end{array}\right]
$$

ТЕОРема 3. Пусть даны две обобщенные интерполячионнье задачи $\mathscr{P}^{(1)}$ и $\mathscr{P}^{(2)}$, причем $\mathscr{P}^{(1)} \subset \mathscr{P}^{(2)}$. Пусть $U_{1}^{(k)}, U_{2}^{(k)}, \mathscr{Z}^{(k)}, \mathscr{F}^{(k)}, s_{F}^{(k)}, s_{K}^{(k)}$ обозначают определенные выше обгекты для интерполяционных задач $\mathscr{P}^{(k)}, k=1,2$. Тогда справедливо следующее.

1. Имеют место неравенства

$$
\begin{gathered}
\frac{J-U_{r}^{(2)}(z) J U_{r}^{(2)^{*}}(z)}{i(z-\bar{z})} \geqslant \frac{J-U_{r}^{(1)}(z) J U_{r}^{(1)^{*}}(z)}{i(z-\bar{z})}, \\
\frac{U_{r}^{(2)^{-1^{*}}}(z) J U_{r}^{(2)^{-1}}(z)}{i(\bar{z}-z)} \leqslant \frac{U_{r}^{(1)^{-1^{*}}}(z) J U_{r}^{(1)^{-1}}(z)}{i(\bar{z}-z)}, \\
r=1,2, \quad \forall z \in \mathbb{C}_{ \pm} \backslash\left\{\mathscr{Z}(1) \cup \mathscr{Z}^{(2)} \cup \overline{\mathscr{Z}}(1) \cup \overline{\mathscr{Z}}(2)\right\} ; \\
U_{1}^{(2)}(z) J_{\pi} U_{1}^{(2)^{*}}(z)-J_{\pi} \geqslant U_{1}^{(1)}(z) J_{\pi} U_{1}^{(1)^{*}}(z)-J_{\pi} \\
\forall z \in \Pi_{-} \backslash\left\{\overline{\mathscr{Z}}^{(1)} \cup \overline{\mathscr{Z}}^{(2)}\right\} .
\end{gathered}
$$

2. Множество решений задачи $\mathscr{P}^{(2)}$ содержится в множестве решений задачи $\mathscr{P}^{(1)}$ :

$$
\mathscr{F}^{(2)} \subset \mathscr{F}^{(1)}
$$

3. Имеют место неравенства

$$
0<s_{F}^{(1)}(x) \leqslant s_{F}^{(2)}(x) \leqslant s_{K}^{(2)}(x) \leqslant s_{K}^{(1)}(x) \quad \forall x \in \mathbb{R}_{-} .
$$

Более того, $s_{F}^{(2)}(x)<s_{K}^{(2)}(x) \quad \forall x \in \mathbb{R}_{-} \backslash\left\{\mathscr{Z}^{(1)} \cup \mathscr{Z}^{(2)}\right\}$.

В терминах интервалов Вейля последние неравенства можно записать в виде $\mathscr{I}^{(2)}(x) \subset \mathscr{I}^{(1)}(x)$. Причем эти оба интервала невырождены во всех mочках $x \in \mathbb{R}_{-} \backslash\left\{\mathscr{Z}^{(1)} \cup \mathscr{Z}^{(2)}\right\}$. 
ДокАЗАТЕЛЬСтво. Сделаем предварительные замечания. Из условия теоремы следует, что масштабные пространства $\mathscr{G}_{1}^{(2)}$ и $\mathscr{G}_{2}^{(2)}$ интерполяционной задачи $\mathscr{P}^{(2)}$ представимы в виде

$$
\mathscr{G}_{r}^{(2)}=\widetilde{\mathscr{G}}_{r}^{(2)} \oplus \widehat{\mathscr{G}}_{r}^{(2)}, \quad r=1,2,
$$

так что выполнены условия (32) и сужение интерполяционной задачи $\mathscr{P}(2)$ на подпространства $\widetilde{\mathscr{G}}_{r}^{(2)}$, обозначаемое символом $\widetilde{\mathscr{P}}^{(2)}$, унитарно эквивалентно интерполяционной задаче $\mathscr{P}^{(1)}$. Пусть $\widetilde{U}_{1}^{(2)}, \widetilde{s}_{F}^{(2)}$ обозначают резольвентную матрицу и решение Фридрихса интерполяционной задачи $\widetilde{\mathscr{P}}^{(2)}$. По лемме 2

$$
\widetilde{U}_{1}^{(2)}(z)=U_{1}^{(1)}(z) \quad \forall z \in \mathbb{C} \backslash\left\{\mathbb{R}_{+} \cup \overline{\mathscr{Z}}\right\}, \quad \widetilde{s}_{F}^{(2)}(x)=s_{F}^{(1)}(x) \quad \forall x \in \mathbb{R}_{-} .
$$

Теперь приступим к доказательству теоремы.

1. Имеем (для упрошения нижеследующих выражений мы отождествляем запись операторов в пространствах $\mathscr{G}_{r}^{(2)}$ с их матричными представлениями как операторов в пространствах $\left.\widetilde{\mathscr{G}}_{r}^{(2)} \oplus \widehat{\mathscr{G}}_{r}^{(2)}\right)$

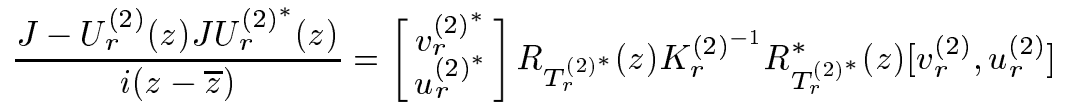

$$
\begin{aligned}
& \geqslant\left[\begin{array}{l}
v_{r}^{(2)^{*}} \\
u_{r}^{(2)^{*}}
\end{array}\right] R_{T_{r}^{(2)^{*}}(z)}\left[\begin{array}{cc}
\widetilde{K}_{r}^{(2)^{-1}} & 0_{\widehat{\mathscr{G}}_{r}^{(2)} \widetilde{\mathscr{G}}_{r}^{(2)}} \\
0_{\widetilde{\mathscr{G}}_{r}^{(2)} \widehat{\mathscr{G}}_{r}^{(2)}} & 0_{\widehat{\mathscr{G}}_{r}^{(2)}}^{(2)}
\end{array}\right] R_{T_{r}^{(2) *}}^{*}(z)\left[v_{r}^{(2)}, u_{r}^{(2)}\right] \\
& =\left[\begin{array}{l}
v_{r}^{(2)^{*}} \\
u_{r}^{(2)^{*}}
\end{array}\right]\left[\begin{array}{cc}
I_{\widetilde{\mathscr{G}}_{r}^{(2)}} & 0_{\widehat{\mathscr{G}}_{r}^{(2)} \widetilde{\mathscr{G}}_{r}^{(2)}} \\
0_{\widetilde{\mathscr{G}}_{r}^{(2)} \widehat{\mathscr{G}}_{r}^{(2)}} & 0_{\widehat{\mathscr{G}}_{r}^{(2)}}
\end{array}\right] R_{T_{r}^{(2) *}}(z)\left[\begin{array}{cc}
I_{\widetilde{\mathscr{G}}_{r}^{(2)}} & 0_{\widehat{\mathscr{G}}_{r}^{(2)} \widetilde{\mathscr{G}}_{r}^{(2)}} \\
0_{\widetilde{\mathscr{G}}_{r}^{(2)} \widehat{\mathscr{G}}_{r}^{(2)}} & 0_{\widehat{\mathscr{G}}_{r}^{(2)}}
\end{array}\right] \\
& \times\left[\begin{array}{cc}
\widetilde{K}_{r}^{(2)^{-1}} & 0_{\widehat{\mathscr{G}}_{r}^{(2)} \widetilde{\mathscr{G}}_{r}^{(2)}} \\
0_{\widetilde{\mathscr{G}}_{r}^{(2)} \widehat{\mathscr{G}}_{r}^{(2)}} & 0_{\widehat{\mathscr{G}}_{r}^{(2)}}
\end{array}\right]\left[\begin{array}{cc}
I_{\widetilde{\mathscr{G}}_{r}^{(2)}} & 0_{\widehat{\mathscr{G}}_{r}^{(2)} \widetilde{\mathscr{G}}_{r}^{(2)}} \\
0_{\widetilde{\mathscr{G}}_{r}^{(2)} \widehat{\mathscr{G}}_{r}^{(2)}} & 0_{\widehat{\mathscr{G}}_{r}^{(2)}}
\end{array}\right] \\
& \times R_{T_{r}^{(2) *}}^{*}(z)\left[\begin{array}{cc}
I_{\widetilde{\mathscr{G}}_{r}^{(2)}} & 0_{\widehat{\mathscr{G}}_{r}^{(2)} \widetilde{\mathscr{G}}_{r}^{(2)}} \\
0_{\widetilde{\mathfrak{G}}_{r}^{(2)} \widehat{\mathscr{G}}_{r}^{(2)}} & 0_{\widehat{\mathscr{G}}_{r}^{(2)}}^{(2)}
\end{array}\right]\left[v_{r}^{(2)}, u_{r}^{(2)}\right]
\end{aligned}
$$

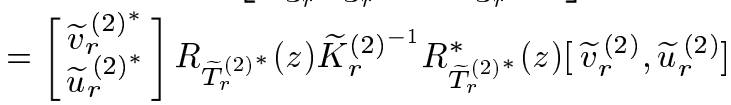

$$
\begin{aligned}
& =\frac{J-\widetilde{U}_{r}^{(2)}(z) J \widetilde{U}_{r}^{(2)^{*}}(z)}{i(z-\bar{z})}=\frac{J-U_{r}^{(1)}(z) J U_{r}^{(1)^{*}}(z)}{i(z-\bar{z})} .
\end{aligned}
$$

В этой цепочке первое равенства следует из (10), неравенство - из (36), второе и третье равенства - из (33), четвертое равенство - из определения интерполяционной задачи $\widetilde{\mathscr{P}}^{(2)}$, пятое - из (41). Остальные неравенства в $(37)$ и (38) доказываются аналогичным образом.

2. Пусть $s \in \mathscr{F}^{(2)}$. Тогда для $s$ выполнена система ОМН В. П. Потапова (13). Воспользовавшись (37), получим $(r=1,2)$

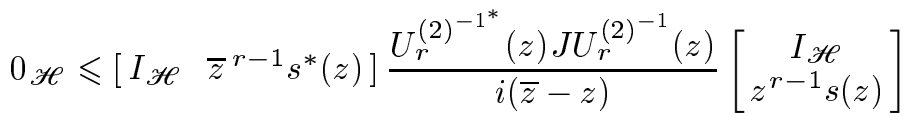

$$
\begin{aligned}
& \leqslant\left[I_{\mathscr{H}} \bar{z}^{r-1} s^{*}(z)\right] \frac{U_{r}^{(1)^{-1^{*}}}(z) J U_{r}^{(1)^{-1}}(z)}{i(\bar{z}-z)}\left[\begin{array}{c}
I_{\mathscr{H}} \\
z^{r-1} s(z)
\end{array}\right] \\
& \forall z \in \mathbb{C}_{ \pm} \backslash\left\{\mathscr{Z}^{(1)} \cup \mathscr{Z}^{(2)}\right\} .
\end{aligned}
$$


Поэтому $s \in \mathscr{F}^{(1)}$. Таким образом, $\mathscr{F}^{(2)} \subset \mathscr{F}^{(1)}$.

3. В теореме 2 доказано, что $s_{F}^{(2)}(x) \leqslant s_{K}^{(2)}(x) \forall x \in \mathbb{R}_{-}$и $s_{F}^{(2)}(x)<s_{K}^{(2)}(x)$ $\forall x \in \mathbb{R}_{-} \backslash\left\{\mathscr{Z}^{(1)} \cup \mathscr{Z}^{(2)}\right\}$.

Далее, $s_{F}^{(2)}, s_{K}^{(2)}(x) \in \mathscr{F}^{(2)} \subset \mathscr{F}^{(1)}$. По теореме 2 для всех $x \in \mathbb{R}_{-}$имеем $s_{F}^{(1)}(x) \leqslant s_{F}^{(2)}(x)$ и $s_{K}^{(2)}(x) \leqslant s_{K}^{(1)}(x)$. Неравенство $0<s_{F}^{(1)}(x)$ следует из формулы (16) и условий полной неопределенности (8).

\section{§4. Предельная интерполяционная задача}

Пусть символ $\mathscr{L}$ обозначает произвольное непустое ограниченное сверху подмножество в множестве вешественных чисел и $A$ - его точная верхняя грань (случай $A=+\infty$ не исключается). И пусть в любой левой полуокрестности $A$ имеется бесконечное множество элементов из $\mathscr{L}$. Множество $\mathscr{L}$ назовем множеством индексов, а его элементы будем обозначать так: $l \in \mathscr{L}$.

ОПРЕДЕЛЕНИЕ 11. Говорят, что задано упорядоченное семейство интерполяционных задач стилтьесовского типа, если каждому индексу $l \in \mathscr{L}$ поставлена в соответствие обобщенная интерполяционная задача стилтьесовского типа $\mathscr{P}^{(l)}$ так, что для любых индексов $l_{1} \leqslant l_{2}$ выполнено условие $\mathscr{P}^{\left(l_{1}\right)} \subset \mathscr{P}^{\left(l_{2}\right)}$.

Упорядоченное семейство интерполяционных задач обозначим через $\left\{\mathscr{P}^{(l)}\right\}_{l \in \mathscr{L}}$. В этом контексте обобщенные интерполяционные задачи $\mathscr{P}^{(l)}$ называются усеченныцми интерполяционными задачами.

Теперь мы исследуем свойства введенных выше объектов при изменяющихся $l$. Для этого в обозначения объектов введем верхний индекс $(l)$, который будет указывать, что соответствуюшие интервалы Вейля, резольвентные матрицы и т.д. связаны с $l$-й интерполяционной задачей.

ОПРЕДЕЛЕНИЕ 12. Пусть дано упорядоченное семейство интерполяционных задач $\left\{\mathscr{P}^{(l)}\right\}_{l \in \mathscr{L}}$, и пусть $\mathscr{F}^{(l)}$ обозначает множество всех решений интерполяци-

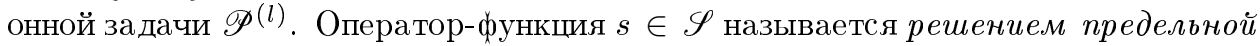
интерполячионной задачи, если $s \in \mathscr{F}(l) \forall l \in \mathscr{L}$.

Множество решений предельной интерполяционной задачи обозначим символом $\mathscr{F}^{(\infty)}$, а саму предельную интерполяционную задачу - символом $\mathscr{P}(\infty)$.

Пусть $A \in\{\mathscr{H}\}$. Из свойств скалярного произведения и линейности оператора $A$ следует поляризационное тождество

$$
\begin{aligned}
4(f, A h)= & (f+h, A(f+h))-(f-h, A(f-h))-i(f+i h, A(f+i h)) \\
& +i(f-i h, A(f-i h)) \quad \forall f, h \in \mathscr{H} .
\end{aligned}
$$

ЛЕмма 3. Пусть для семейства стилтьесовских о. $\phi .\left\{s^{(l)}\right\}_{l \in \mathscr{L}}$ существует вещественная константа $C>0$ такая, что в некоторой точке $x_{0} \in \mathbb{R}_{-}$ виполняется неравенство $\left\|s^{(l)}\left(x_{0}\right)\right\| \leqslant C \quad \forall l \in \mathscr{L}$.

Тогда для любого компакта $K \subset \mathbb{C} \backslash \mathbb{R}_{+}$существует вещественная константа $C(K)>0$ такая, что $\left\|s^{(l)}(z)\right\| \leqslant C(K) \quad \forall l \in \mathscr{L}, \forall z \in K$. 
ДокАЗАТЕльство. Зафиксируем вектор $f \in \mathscr{H}$ и рассмотрим функции

$$
\left\{s_{f}^{(l)}(z):=\left(f, s^{(l)}(z) f\right), z \in \mathbb{C} \backslash \mathbb{R}_{+}\right\}_{l \in \mathscr{L}} .
$$

Это скалярные стилтьесовские функции, и они ограничены в точке $x_{0}$. Из представления (6) следует, что $s_{f}^{(l)}(z)+s_{f}^{(l) *}(z) \geqslant 0, z \in \Pi_{-}$. Поэтому каждая из функций $w^{(l)}(z):=i s_{f}^{(l)}(i z), z \in \mathbb{C}_{+}, l \in \mathscr{L}$, является неванлинновской и эти функции ограничены в точке $-i x_{0} \in \mathbb{C}_{+}$. Но тогда (см. [20; $\S 2$, лемма 3$\left.]\right)$ семейство неванлинновских функций $\left\{w^{(l)}\right\}_{l \in \mathscr{L}}$ равномерно ограничено на компактах, содержашихся в $\mathbb{C}_{+}$. Отсюда и из определения функций $w^{(l)}$ следует, что семейство функций $\left\{s_{f}^{(l)}\right\}_{l \in \mathscr{L}}$ равномерно ограничено на компактах из П_.

Пусть некоторая точка $z_{0} \in \mathbb{C}_{+} \cap \Pi_{-}$. По уже доказанному семейство неванлинновских функций $\left\{s_{f}^{(l)}(z), z \in \mathbb{C}_{+}\right\}_{l \in \mathscr{L}}$ ограничено в точке $z_{0}$. Но тогда (см. [20; $\S 2$, лемма 3]) это семейство функций равномерно ограничено на компактах из $\mathbb{C}_{+}$. Из принципа симметрии $s_{f}^{(l)}(z)=\overline{s_{f}^{(l)}(\bar{z})}$ следует, что семейство функций $\left\{s_{f}^{(l)}(z), z \in \mathbb{C}_{-}\right\}_{l \in \mathscr{L}}$ равномерно ограничено на компактах из $\mathbb{C}_{-}$.

Семейство функций $\left\{s_{f}^{(l)}(z), z \in \mathbb{C} \backslash \mathbb{R}_{+}\right\}_{l \in \mathscr{L}}$ равномерно ограничено на компактах из полуплоскостей $\mathbb{C}_{+}, \mathbb{C}_{-}$и П_. Поэтому оно равномерно ограничено и на компактах, содержащихся в $\mathbb{C} \backslash \mathbb{R}_{+}$.

Итак, при любом векторе $f \in \mathscr{H}$ семейство функций $s_{f}^{(l)}(z):=\left(f, s^{(l)}(z) f\right)$, $z \in \mathbb{C} \backslash \mathbb{R}_{+}, l \in \mathscr{L}$, равномерно ограничено на компактах, содержашихся в $\mathbb{C} \backslash \mathbb{R}_{+}$. Но тогда в силу поляризационного тождества (42) на компактах, содержашихся в $\mathbb{C} \backslash \mathbb{R}_{+}$, равномерно ограничено семейство $\left\{s_{g f}^{(l)}(z):=\left(g, s^{(l)}(z) f\right), z \in \mathbb{C} \backslash \mathbb{R}_{+},\right\}_{l \in \mathscr{L}}$ при любых $f, g \in \mathscr{H}$. Отсюда следует, что семейство о.ф. $\left\{s^{(l)}\right\}_{l \in \mathscr{L}}$ по норме равномерно ограничено на компактах, содержащихся в $\mathbb{C} \backslash \mathbb{R}_{+}$.

Лемма 4. Пусть семейство стилтьесовских о. $\phi .\left\{s^{(l)}\right\}_{l \in \mathscr{L}}$ таково, что существуют предель $\lim _{l \rightarrow A} s^{(l)}(x) \quad \forall x \in \mathbb{R}_{-}$.

Тогда существуют равномерные на компактах из $\mathbb{C} \backslash \mathbb{R}_{+}$предель

$$
s^{(\infty)}(z):=\lim _{l \rightarrow A} s^{(l)}(z) \quad \forall z \in \mathbb{C} \backslash \mathbb{R}_{+} \quad u \quad s^{(\infty)} \in \mathscr{S} .
$$

ДоказАТЕЛЬСТво. Пусть $K$ - произвольньй компакт из $\mathbb{C} \backslash \mathbb{R}_{+}$. Обозначим через $\Omega$ односвязную открытую область с гладкой границей такую, что

a) $K \subset \Omega$,

b) замыкание $\Omega$ является компактом в $\mathbb{C} \backslash \mathbb{R}_{+}$,

c) область $\Omega$ содержит некоторый интервал $(a, b) \subset \mathbb{R}_{-}$.

В любой точке $x_{0} \in \mathbb{R}_{-}$семейство операторов $\left\{s_{M}^{(l)}\left(x_{0}\right)\right\}_{l \in \mathscr{L}}$ сходится при $l \rightarrow A$ и, следовательно, ограничено по норме. По лемме 4 семейство о.ф. $\left\{s_{M}^{(l)}\right\}_{l \in \mathscr{L} \text { рав- }}$ номерно ограничено в замыкании области $\Omega$. Семейство операторов $\left\{s_{M}^{(l)}(x)\right\}_{l \in \mathscr{L}}$ сходится $\forall x \in(a, b)$. По теореме Витали семейство о.ф. $\left\{s_{M}^{(l)}\right\}_{l \in \mathscr{L}}$ равномерно сходится при $z \in K$. Ясно, что предельная о.ф.. $s^{(\infty)} \in \mathscr{\mathscr { S }}$. 
ТЕОРема 4. Пусть задано упорядоченное семейство обобщенных интерполяиионных задач стилтьесовского типа $\left\{\mathscr{P}^{(l)}\right\}_{l \in \mathscr{L}}$, и пусть $\mathscr{F}^{(l)}$ обозначает множество решений задачи $\mathscr{P}^{(l)}$, а $\mathscr{F}(\infty)$ - множество решений соответствующей предельной интерполяционной задачи. И пусть, далее, $s_{F}^{(l)} u s_{K}^{(l)}$ являются главньими решениями задачи $\mathscr{P}^{(l)}$. Тогда справедливо следующее.

1. Существуют равномерные на компактах $K \subset \mathbb{C} \backslash \mathbb{R}_{+}$предель

$$
s_{K}^{(\infty)}(z):=\lim _{l \rightarrow A} s_{K}^{(l)}(z) \in \mathscr{F}^{(\infty)}, \quad s_{F}^{(\infty)}(z):=\lim _{l \rightarrow A} s_{F}^{(l)}(z) \in \mathscr{F}^{(\infty)} .
$$

2. Для всех $s \in \mathscr{F}(\infty)$ виполняются неравенства

$$
0 \mathscr{H}<s_{F}^{(\infty)}(x) \leqslant s(x) \leqslant s_{K}^{(\infty)}(x) \quad \forall x \in \mathbb{R}_{-} .
$$

ДокАЗАТЕльство. 1. Зафиксируем некоторый индекс $j \in \mathscr{L}$. Из неравенств (40) следует, что $\forall x \in \mathbb{R}_{-}$

$$
s_{F}^{\left(l_{1}\right)}(x) \leqslant s_{F}^{\left(l_{2}\right)}(x) \leqslant s_{K}^{(j)}(x), \quad l_{1}, l_{2} \in \mathscr{L}, \quad l_{1} \leqslant l_{2} \leqslant j .
$$

Поэтому в каждой точке $x \in \mathbb{R}_{-}$семейство эрмитовых операторов $\left\{s_{F}^{(l)}(x)\right\}_{l \in \mathscr{L}}$ монотонно возрастает и ограничено сверху оператором $s_{K}^{(j)}(x)$. Отсюда следует, что существует предел $\lim _{l \rightarrow A} s_{F}^{(l)}(x) \forall x \in \mathbb{R}_{-}$. По лемме 4 отсюда следует существование первого из пределов в (44). Аналогично доказывается существование второго предела в (44).

Пусть зафиксированы два индекса $l_{1}, l \in \mathscr{L}$, и пусть $l_{1}<l$. По теореме 3 о.ф. $s_{F}^{(l)} \in \mathscr{F}^{\left(l_{1}\right)}$ и, следовательно, выполнена система ОМН В. П. Потапова $(r=1,2)$ :

$$
\left[I_{\mathscr{H}} \quad \bar{z}^{r-1} s_{F}^{(l)^{*}}(z)\right] \frac{U_{r}^{\left(l_{1}\right)^{-1^{*}}}(z) J U_{r}^{\left(l_{1}\right)^{-1}}(z)}{i(\bar{z}-z)}\left[\begin{array}{c}
I_{\mathscr{H}} \\
z^{r-1} s_{F}^{(l)}(z)
\end{array}\right] \geqslant 0 \mathscr{H}, \quad z \in \mathbb{C}_{ \pm} \backslash \mathscr{Z}^{\left(l_{1}\right)} .
$$

Перейдем в этих неравенствах к пределу $l \rightarrow A$. Получим, что $s_{F}^{(\infty)} \in \mathscr{F}^{\left(l_{1}\right)}$. В силу произвольности $l_{1}$ отсюда следует, что $s_{F}^{(\infty)} \in \mathscr{F}(\infty)$. Утверждение относительно $s_{K}^{(\infty)}$ доказьвается аналогичным образом.

2. Пусть задана некоторая о.ф. $s \in \mathscr{F}(\infty)$. По определению $s \in \mathscr{F}(l) \forall l \in \mathscr{L}$. По теореме 2 имеют место неравенства $0_{\mathscr{C}}<s_{F}^{(l)}(x) \leqslant s(x) \leqslant s_{K}^{(l)}(x)$. Переходя к пределу $l \rightarrow A$, получим (45). При этом первое из неравенств остается строгим неравенством и после предельного перехода в силу монотонного возрастания по $l$ семейства операторов $s_{F}^{(l)}(x)$.

Доказанная теорема прежде всего указывает на то, что множество решений предельной интерполяционной задачи непусто. Например, $s_{F}^{(\infty)}, s_{K}^{(\infty)} \in \mathscr{F}(\infty)$. Из неравенств (45) следует, что решения предельной интерполяционной задачи $s_{F}^{(\infty)}, s_{K}^{(\infty)}$ для множества $\mathscr{F}(\infty)$ играют такую же роль, какую решения $s_{F}^{(l)}, s_{K}^{(l)}$ играют для множества $\mathscr{F}^{(l)}$. Поэтому представляется естественным назвать $s_{F}^{(\infty)}$ и $s_{K}^{(\infty)}$ главными решениями Фридрихса и Крейна предельной интерполяционной 
задачи. Если решения Фридрихса и Крейна предельной интерполяционной задачи совпадают, то они задают единственное решение предельной интерполяционной задачи. Это следует из неравенств (45). В противном случае предельная интерполяционная задача имеет бесконечно много решений. Действительно, пусть заданы два числа $p, q \in \mathbb{R}_{+}$такие, что $p+q=1$. Тогда о.ф. $s(z)=p s_{F}^{(\infty)}(z)+$ $q s_{K}^{(\infty)}(z) \in \mathscr{F}^{(l)} \forall l \in \mathscr{L}$ (см. вид системы ОМН (7)). Таким образом, $s \in \mathscr{F}^{(\infty)}$. По аналогии с определением 7 дадим определение предельных интервалов Вейля.

ОПРЕДЕЛЕНИЕ 13. Операторный интервал

$$
\mathscr{I}^{(\infty)}(x):=\left[s_{F}^{(\infty)}(x), s_{K}^{(\infty)}(x)\right], \quad x \in \mathbb{R}_{-},
$$

называется предельным интервалом Вейля в точке $x \in \mathbb{R}_{-}$.

Из (45) следует включение $\{s(x): s \in \mathscr{F}(\infty)\} \subset \mathscr{I}^{(\infty)}(x)$.

\section{§5. Критерий неопределенности}

ЛЕмма 5. Пусть дано упорядоченное семейство интерполяиионных задач

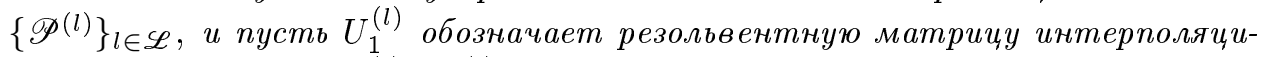
онной задачи $\mathscr{P}^{(l)}$, а $s_{F}^{(l)}$ и $s_{K}^{(l)}$ - соответствующие главнье решения.

Тогда для всех $x \in \mathbb{R}_{-} \backslash \mathscr{Z}^{(l)}$ имеем

$$
\begin{aligned}
& U_{1}^{(l)}(x) J_{\pi} U_{1}^{(l) *}(x)-J_{\pi} \\
& \quad=2\left[\begin{array}{cc}
I_{\mathscr{H}} & 0 \mathscr{H} \\
s_{F}^{(l)}(x) & I_{\mathscr{H}}
\end{array}\right]\left[\begin{array}{cc}
\left(s_{K}^{(l)}(x)-s_{F}^{(l)}(l)\right)^{-1} & 0_{\mathscr{H}} \\
0 \mathscr{H} & s_{F}^{(l)}(x)
\end{array}\right]\left[\begin{array}{cc}
I_{\mathscr{H}} & s_{F}^{(l)}(x) \\
0_{\mathscr{H}} & I_{\mathscr{H}}
\end{array}\right] .
\end{aligned}
$$

ДокАЗАТЕЛЬство. Для упрощения записи верхний индекс $(l)$ опускаем.

$$
\begin{aligned}
U_{1}(x) & =\left[\begin{array}{cc}
\alpha(x) & \beta(x) \\
\gamma(x) & \delta(x)
\end{array}\right]=\left[\begin{array}{cc}
I_{\mathscr{H}} & I_{\mathscr{H}} \\
\gamma(x) \alpha^{-1}(x) & \delta(x) \beta^{-1}(x)
\end{array}\right]\left[\begin{array}{cc}
\alpha(x) & 0 \mathscr{H} \\
0 \mathscr{H} & \beta(x)
\end{array}\right] \\
& =\left[\begin{array}{cc}
I_{\mathscr{H}} & I_{\mathscr{H}} \\
s_{F}(x) & s_{K}(x)
\end{array}\right]\left[\begin{array}{cc}
\alpha(x) \beta^{*}(x) & 0 \mathscr{H} \\
0 \mathscr{H} & I_{\mathscr{H}}
\end{array}\right]\left[\begin{array}{cc}
\beta^{*^{-1}}(x) & 0 \mathscr{H} \\
0_{\mathscr{H}} & \beta(x)
\end{array}\right] \\
& =\left[\begin{array}{cc}
I_{\mathscr{H}} & I_{\mathscr{H}} \\
s_{F}(x) & s_{K}(x)
\end{array}\right]\left[\begin{array}{cc}
\left(s_{K}(x)-s_{F}(x)\right)^{-1} & 0_{\mathscr{H}} \\
0_{\mathscr{H}} & I_{\mathscr{H}}
\end{array}\right]\left[\begin{array}{cc}
\beta^{*^{-1}}(x) & 0 \mathscr{H} \\
0_{\mathscr{H}} & \beta(x)
\end{array}\right] .
\end{aligned}
$$

В этой цепочке равенств третье следует из (15), а четвертое - из (21) и эрмитовости $s_{K}(x), s_{F}(x)$. Далее (для упрошения обозначений опускаем аргумент $x$ ),

$$
\begin{aligned}
& U_{1} J_{\pi} U_{1}^{*}-J_{\pi} \\
& =\left[\begin{array}{cc}
I_{\mathscr{H}} & I_{\mathscr{H}} \\
s_{F} & s_{K}
\end{array}\right]\left[\begin{array}{cc}
\left(s_{K}-s_{F}\right)^{-1} & 0_{\mathscr{H}} \\
0_{\mathscr{H}} & I_{\mathscr{H}}
\end{array}\right]\left[\begin{array}{cc}
\beta^{*^{-1}} & 0_{\mathscr{H}} \\
0_{\mathscr{H}} & \beta
\end{array}\right] J_{\pi} \\
& \times\left[\begin{array}{cc}
\beta^{-1} & 0_{\mathscr{H}} \\
0 \mathscr{H} & \beta^{*}
\end{array}\right]\left[\begin{array}{cc}
\left(s_{K}-s_{F}\right)^{-1} & 0_{\mathscr{H}} \\
0 \mathscr{H} & I_{\mathscr{H}}
\end{array}\right]\left[\begin{array}{cc}
I_{\mathscr{H}} & s_{F} \\
I_{\mathscr{H}} & s_{K}
\end{array}\right]-J_{\pi} \\
& =\left[\begin{array}{cc}
I_{\mathscr{H}} & I_{\mathscr{H}} \\
s_{F} & s_{K}
\end{array}\right]\left[\begin{array}{cc}
\left(s_{K}-s_{F}\right)^{-1} & 0_{\mathscr{H}} \\
0 \mathscr{H} & I_{\mathscr{H}}
\end{array}\right] J_{\pi}\left[\begin{array}{cc}
\left(s_{K}-s_{F}\right)^{-1} & 0_{\mathscr{H}} \\
0 \mathscr{H} & I_{\mathscr{H}}
\end{array}\right]\left[\begin{array}{cc}
I_{\mathscr{H}} & s_{F} \\
I_{\mathscr{H}} & s_{K}
\end{array}\right]-J_{\pi}
\end{aligned}
$$




$$
\begin{aligned}
& =\left[\begin{array}{cc}
2\left(s_{K}-s_{F}\right)^{-1} & 2\left(s_{K}-s_{F}\right)^{-1} s_{F} \\
2 s_{F}\left(s_{K}-s_{F}\right)^{-1} & s_{F}\left(s_{K}-s_{F}\right)^{-1} s_{K}+s_{K}\left(s_{K}-s_{F}\right)^{-1} s_{F}
\end{array}\right] \\
& =2\left[\begin{array}{cc}
\left(s_{K}-s_{F}\right)^{-1} & \left(s_{K}-s_{F}\right)^{-1} s_{F} \\
s_{F}\left(s_{K}-s_{F}\right)^{-1} & \left(s_{F}^{-1}-s_{K}^{-1}\right)^{-1}
\end{array}\right] \\
& =2\left[\begin{array}{cc}
\left(s_{K}-s_{F}\right)^{-1} & \left(s_{K}-s_{F}\right)^{-1} s_{F} \\
s_{F}\left(s_{K}-s_{F}\right)^{-1} & s_{F}\left(s_{K}-s_{F}\right)^{-1} s_{K}
\end{array}\right] \\
& =2\left[\begin{array}{cc}
\left(s_{K}-s_{F}\right)^{-1} & \left(s_{K}-s_{F}\right)^{-1} s_{F} \\
s_{F}\left(s_{K}-s_{F}\right)^{-1} & s_{F}\left(s_{K}-s_{F}\right)^{-1} s_{F}+s_{F}
\end{array}\right] \\
& =2\left[\begin{array}{cc}
I_{\mathscr{H}} & 0 \mathscr{H} \\
s_{F} & I_{\mathscr{H}}
\end{array}\right]\left[\begin{array}{cc}
\left(s_{K}-s_{F}\right)^{-1} & 0 \mathscr{H} \\
0 \mathscr{H} & s_{F}
\end{array}\right]\left[\begin{array}{cc}
I_{\mathscr{H}} & s_{F} \\
0 \mathscr{H} & I_{\mathscr{H}}
\end{array}\right] .
\end{aligned}
$$

Пусть индекс $l \in \mathscr{L}$. Напомним, что множество особых точек мероморфных о.ф. $R_{T_{1}^{(l)}}$ и $R_{T_{2}^{(l)}}$ в $\mathbb{C} \backslash \mathbb{R}_{+}$мы обозначили символом $\mathscr{Z}^{(l)}$. Пусть $\mathscr{Z}(\infty):=\bigcup_{l \in \mathscr{L}} \mathscr{Z}^{(l)}$. С этого места и до конца статьи мы будем считать, что все предельные точки $\mathscr{Z}(\infty)$ принадлежат множеству $\mathbb{R}_{+} \cup\{\infty\}$. Смысл этого условия состоит в том, что наличие предельной точки множества $\mathscr{Z}(\infty)$ в области $\mathbb{C} \backslash \mathbb{R}_{+}$приводит к вырождению предельной интерполяционной задачи. Нас же будет интересовать невырожденньй случай.

ЛЕмма 6. Пусть дано упорядоченное семейство интерполяиионных задач

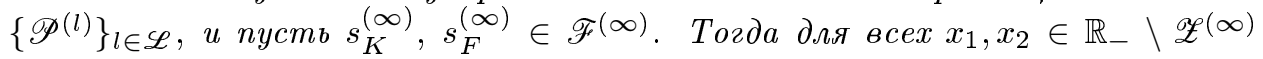
имеет место равенство

$$
\operatorname{rank}\left\{s_{K}^{(\infty)}\left(x_{1}\right)-s_{F}^{(\infty)}\left(x_{1}\right)\right\}=\operatorname{rank}\left\{s_{K}^{(\infty)}\left(x_{2}\right)-s_{F}^{(\infty)}\left(x_{2}\right)\right\}
$$

Другими словами, ранги предельных интервалов Вейля $\mathscr{I}^{(\infty)}(x)$ не зависят от въцора точки $x \in \mathbb{R}_{-} \backslash \mathscr{Z}^{(\infty)}$.

ДокАЗАТЕЛЬСтво. Из (38) следует, что $J_{\pi}$ - формы резольвентных матриц монотонно возрастают в левой полуплоскости

$$
U_{1}^{(l)}(z) J_{\pi} U_{1}^{(l)^{*}}(z)-J_{\pi} \geqslant U_{1}^{(k)}(z) J_{\pi} U_{1}^{(k)^{*}}(z)-J_{\pi} \quad \forall z \in \Pi_{-} \backslash \mathscr{Z}^{(\infty)}, l \geqslant k .
$$

Из (12) и (8) имеем

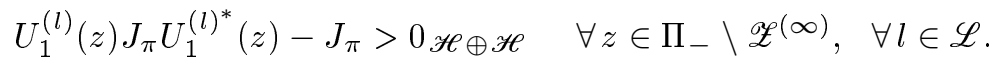

По теореме С.А. Орлова (см. [14], [21]) сушествуют пределы

$$
\Gamma(z):=\lim _{l \rightarrow A}\left[U_{1}^{(l)}(z) J_{\pi} U_{1}^{(l)^{*}}(z)-J_{\pi}\right]^{-1} \quad \forall z \in \Pi_{-} \backslash \mathscr{Z}^{(\infty)}
$$

и для всех $z_{1}, z_{2} \in \Pi_{-} \backslash \mathscr{Z}(\infty)$

$$
\operatorname{rank} \Gamma\left(z_{1}\right)=\operatorname{rank} \Gamma\left(z_{2}\right)
$$

Из (45) следует, что $s_{F}^{(l)}(x)>0 \mathscr{H} \forall l \in \mathscr{L}, \forall x \in \mathbb{R}_{-} \backslash \mathscr{Z}(\infty)$. Отсюда и из (46) имеем

$$
\Gamma(x)=\frac{1}{2}\left[\begin{array}{cc}
I_{\mathscr{H}} & -s_{F}^{(\infty)}(x) \\
0 \mathscr{H} & I_{\mathscr{H}}
\end{array}\right]\left[\begin{array}{cc}
s_{K}^{(\infty)}(x)-s_{F}^{(\infty)}(x) & 0 \mathscr{H} \\
0_{\mathscr{H}} & s_{F}^{(\infty)^{-1}}(x)
\end{array}\right]\left[\begin{array}{cc}
I_{\mathscr{H}} & 0 \mathscr{H} \\
-s_{F}^{(\infty)}(x) & I_{\mathscr{H}}
\end{array}\right]
$$

Следовательно,

$$
\operatorname{rank} \Gamma(x)=\operatorname{rank}\left[s_{K}^{(\infty)}(x)-s_{F}^{(\infty)}(x)\right]+m, \quad m=\operatorname{rank} s_{F}^{(\infty)}(x)=\operatorname{dim} \mathscr{H} .
$$

Отсюда и из (49) следует (48). 
ОПРЕДЕЛЕНиЕ 14. Предельная интерполяционная задача $\mathscr{P}(\infty)$ называется вполне неопределенной, если все предельные интервалы Вейля

$$
\mathscr{I}^{(\infty)}(x)=\left[s_{\mu}^{(\infty)}(x), s_{M}^{(\infty)}(x)\right] \quad \forall x \in \mathbb{R}_{-} \backslash \mathscr{Z}^{(\infty)}
$$

являются невырож денными операторными интервалами.

Из этого определения и леммы 6 непосредственно вытекает

ЛЕМма 7. Для того чтобъ предельная интерполяционная задача $\mathscr{P}(\infty)$ была вполне неопределенной, необходимо, чтобы при всех $x \in \mathbb{R}_{-} \backslash \mathscr{Z}(\infty)$ выполнялось неравенство

$$
s_{F}^{(\infty)}(x)<s_{K}^{(\infty)}(x)
$$

и достаточно, чтобъ неравенство (50) выполнялось хотя бъ для одного $x \in \mathbb{R}_{-} \backslash \mathscr{Z}^{(\infty)}$.

Другими словами, для неопределенности предельной интерполячионной задачи необходимо, чтобь при всех $x \in \mathbb{R}_{-} \backslash \mathscr{Z}(\infty)$ предельные интерваль Вейля были невырождень, и достаточно, чтобъ хотя бъи для одного $x \in$ $\mathbb{R}_{-} \backslash \mathscr{Z}^{(\infty)}$ был невырожсденным соответствующий предельный интервал Вейля.

ТЕОрема 5. Пусть зафиксирована некоторая точка $x_{0} \in \mathbb{R}_{-} \backslash \mathscr{Z}(\infty)$. Для того чтоби предельная интерполячионная задача $\mathscr{P}(\infty)$ была вполне неопределенной, необходимо и достаточно, чтобы существовали строго положсительные предель

$$
\begin{aligned}
& \lim _{l \rightarrow A}\left[v_{1}^{(l)^{*}} R_{T_{1}^{(l)}}^{*}\left(x_{0}\right) K_{1}^{(l)^{-1}} R_{T_{1}^{(l)}}\left(x_{0}\right) v_{1}^{(l)}\right]>0 \mathscr{H} \\
& \lim _{l \rightarrow A}\left[v_{2}^{(l)^{*}} R_{T_{2}^{(l)}}^{*}\left(x_{0}\right) K_{2}^{(l)^{-1}} R_{T_{2}^{(l)}}\left(x_{0}\right) v_{2}^{(l)}\right]>0 \mathscr{H} .
\end{aligned}
$$

ДокАЗАТЕЛЬСтво. Пусть $x_{0} \in \mathbb{R}_{-} \backslash \mathscr{Z}^{(\infty)}$. Из выбора этой точки следует обратимость операторов $R_{T_{r}^{(l)}}\left(x_{0}\right)$. Отсюда и из условий полной неопределенности (8) следует положительная определенность операторов

$$
v_{r}^{(l)^{*}} R_{T_{r}^{(l)}}^{*}\left(x_{0}\right) K_{r}^{(l)^{-1}} R_{T_{r}^{(l)}}\left(x_{0}\right) v_{r}^{(l)} \in\{\mathscr{H}\}_{>}, \quad r=1,2, \quad \forall l \in \mathscr{L} .
$$

Эти операторы монотонно возрастают с ростом $l$ ( в этом можно убедиться с помощь рассуждений, аналогичных приведенньм при доказательстве теоремы 3 ).

Пусть теперь предельная интерполяционная задача $\mathscr{P}(\infty)$ является вполне неопределенной. Для $l$-й обобщенной интерполяционной задачи $\mathscr{P}(l)$ запишем (17) при некотором $z=x_{0} \in \mathbb{R}_{-} \backslash \mathscr{Z}^{(\infty)}$. Получим

$$
\begin{aligned}
\left\{s_{K}^{(l)}\left(x_{0}\right)-s_{F}^{(l)}\left(x_{0}\right)\right\}^{-1}= & -x_{0} v_{1}^{(l)^{*}} R_{T_{1}^{(l)}}^{*}\left(x_{0}\right) K_{1}^{(l)^{-1}} R_{T_{1}^{(l)}}\left(x_{0}\right) v_{1}^{(l)} \\
& +x_{0}^{2} v_{2}^{(l) *} R_{T_{2}^{(l)}}^{*}\left(x_{0}\right) K_{2}^{(l)^{-1}} R_{T_{2}^{(l)}}\left(x_{0}\right) v_{2}^{(l)}
\end{aligned}
$$


Оба слагаемых в правой части последнего равенства являются положительно определенными операторами и монотонно возрастают с ростом $l$. Левая часть в силу неопределенности предельной интерполяционной задачи при $l \rightarrow A$ стремится к положительно определенному оператору $\left\{s_{K}^{(\infty)}\left(x_{0}\right)-s_{F}^{(\infty)}\left(x_{0}\right)\right\}^{-1}$. Отсюда следует существование и строгая положительность пределов в (51).

Наоборот, пусть существуют и строго положительны оба предела в (51). Совершим теперь предельный переход $l \rightarrow A$ в обеих частях равенства (52). Получим, что $\left\{s_{K}^{(\infty)}\left(x_{0}\right)-s_{F}^{(\infty)}\left(x_{0}\right)\right\}^{-1}>0$. Таким образом, предельный интервал Вейля $\mathscr{I}^{(\infty)}\left(x_{0}\right)$ является невырожденньм и, следовательно, предельная интерполяционная задача является вполне неопределенной.

\section{§6. Описание решений предельной задачи}

Пусть дано упорядоченное семейство вполне неопределенных интерполяционных задач $\left\{\mathscr{P}^{(l)}\right\}_{l \in \mathscr{L}}$, и пусть это семейство порождает вполне неопределенную предельную интерполяционную задачу $\mathscr{P}(\infty)$. С каждой усеченной задачей $\mathscr{P}(l)$

мы связали две о.ф. $U_{1}^{(l)}$ и $U_{2}^{(l)}$. Зафиксируем некоторую точку $x_{0} \in \mathbb{R}_{-} \backslash \mathscr{Z}_{\infty}$, и пусть оператор $\beta^{(l)}\left(x_{0}\right)$ является матричным элементом из представления $(9)$ резольвентной матрицы $U_{1}^{(l)}\left(x_{0}\right)$. Рассмотрим оператор

$$
T^{(l)}=\left[\begin{array}{cc}
\beta^{(l)^{*}}\left(x_{0}\right) & 0 \mathscr{H} \\
0_{\mathscr{H}} & \beta^{(l)^{-1}}\left(x_{0}\right)
\end{array}\right] \in\{\mathscr{H} \oplus \mathscr{H}\} .
$$

Теперь введем новую нормировку о.ф. $U_{1}^{(l)}$ и $U_{2}^{(l)}$ :

$$
\widetilde{U}_{1}^{(l)}(z)=U_{1}^{(l)}(z) T^{(l)}, \quad \widetilde{U}_{2}^{(l)}(z)=U_{2}^{(l)}(z) T^{(l)}, \quad z \in \mathbb{C} \backslash\left\{\mathbb{R}_{+} \cup \overline{\mathscr{Z}}_{\infty}\right\} .
$$

Из очевидных равенств

$$
J-T^{(l)} J T^{(l)^{*}}=0 \mathscr{H} \oplus \mathscr{H}, \quad J_{\pi}-T^{(l)} J_{\pi} T^{(l)^{*}}=0 \mathscr{H} \oplus \mathscr{H}
$$

следует, что во всех наших рассуждениях можно заменить о.ф. $U_{1}^{(l)}$ и $U_{2}^{(l)}$ на нормированные о.ф. $\widetilde{U}_{1}^{(l)}$ и $\widetilde{U}_{2}^{(l)}$. В точке $x_{0}($ см. $(47))$ имеем

$$
\widetilde{U}_{1}^{(l)}\left(x_{0}\right)=\left[\begin{array}{cc}
I_{\mathscr{H}} & I_{\mathscr{H}} \\
s_{F}^{(l)}\left(x_{0}\right) & s_{K}^{(l)}\left(x_{0}\right)
\end{array}\right]\left[\begin{array}{cc}
\left(s_{K}^{(l)}\left(x_{0}\right)-s_{F}^{(l)}\left(x_{0}\right)\right)^{-1} & 0_{\mathscr{H}} \\
0 \mathscr{H} & I_{\mathscr{H}}
\end{array}\right] .
$$

ТЕОРема 6. Пусть дано упорядоченное семейство вполне неопределен-

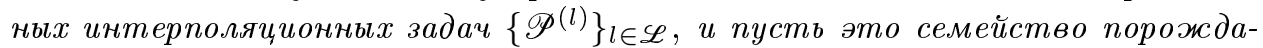
ет вполне неопределенную предельную интерполяционную задачу $\mathscr{P}(\infty)$. пусть, далее, дана некоторая точка $x_{0} \in \mathbb{R}_{-} \backslash \mathscr{Z}^{(\infty)}$ и нормированные резольвентные о.ф. усеченных задач $\widetilde{U}_{1}^{(l)}$ и $\widetilde{U}_{2}^{(l)}$ определены формулами (53). 
Тогда существуют равномерные предель на компактах $K \subset \mathbb{C} \backslash\left\{\mathbb{R}_{+} \cup\right.$ $\left.\mathscr{Z}^{(\infty)} \cup \overline{\mathscr{Z}}(\infty)\right\}:$

$$
\begin{aligned}
U_{1}^{(\infty)}(z) & :=\lim _{l \rightarrow A} \widetilde{U}_{1}^{(l)}(z), & U_{2}^{(\infty)}(z) & :=\lim _{l \rightarrow A} \widetilde{U}_{2}^{(l)}(z), \\
U_{1}^{(\infty)^{-1}}(z) & :=\lim _{l \rightarrow A} \widetilde{U}_{1}^{(l)^{-1}}(z), & U_{2}^{(\infty)^{-1}}(z) & :=\lim _{l \rightarrow A} \widetilde{U}_{2}^{(l)}{ }^{-1}(z),
\end{aligned}
$$

и о.ф. $U_{1}^{(\infty)}(z), U_{2}^{(\infty)}(z), U_{1}^{(\infty)^{-1}}(z)$ и $U_{2}^{(\infty)^{-1}}(z)$ являются голоморфными при $z \in \mathbb{C} \backslash\left\{\mathbb{R}_{+} \cup \mathscr{Z}^{(\infty)} \cup \overline{\mathscr{Z}}(\infty)\right\}$.

ДокАЗАТЕльство. Мы предположили, что предельные точки множества $\mathscr{Z}(\infty)$ принадлежат $\mathbb{R}_{+} \cup\{\infty\}$. Отсюда следует, что в некоторой окрестности точки $x_{0}$ при всех $l \in \mathscr{L}$ голоморфны о.ф. $\widetilde{U}_{1}^{(l)}$. Из неопределенности задачи $\mathscr{P}(\infty)$ и из формулы (55) следует, что семейство операторов $\left\{\widetilde{U}_{1}^{(l)}\left(x_{0}\right)\right\}_{l \in \mathscr{L}}$ сходится при $l \rightarrow A$.

Далее, из $(38),(8)$ и (12) получаем $\left(\forall z \in \Pi_{-} \backslash\{\mathscr{Z}(\infty) \cup \overline{\mathscr{Z}}(\infty)\}\right)$

$$
\begin{aligned}
& U_{1}^{(l)}(z) J_{\pi} U_{1}^{(l)^{*}}(z)-J_{\pi} \geqslant U_{1}^{(j)}(z) J_{\pi} U_{1}^{(j)^{*}}(z)-J_{\pi}, \\
& U_{1}^{(l)}(z) J_{\pi} U_{1}^{(l)^{*}}(z)-J_{\pi}>0 \mathscr{H} \oplus \mathscr{H} \quad \forall l, j \in \mathscr{L}, \quad l \geqslant j .
\end{aligned}
$$

Из (53) и (54) следует, что $J_{\pi}$-формы о.ф. $U_{1}^{(l)}(z)$ и $\widetilde{U}_{1}^{(l)}(z)$ совпадают. Поэтому $\left(\forall z \in \Pi_{-} \backslash\left\{\mathscr{Z}^{(\infty)} \cup \overline{\mathscr{Z}}(\infty)\right\}\right)$

$$
\begin{aligned}
& \widetilde{U}_{1}^{(l)}(z) J_{\pi} \widetilde{U}_{1}^{(l)^{*}}(z)-J_{\pi} \geqslant \widetilde{U}_{1}^{(j)}(z) J_{\pi} \widetilde{U}_{1}^{(j)^{*}}(z)-J_{\pi}, \\
& \widetilde{U}_{1}^{(l)}(z) J_{\pi} \widetilde{U}_{1}^{(l)^{*}}(z)-J_{\pi}>0 \mathscr{H} \oplus \mathscr{H} \quad \forall l, j \in \mathscr{L}, \quad l \geqslant j .
\end{aligned}
$$

Отсюда и из сходимости семейства операторов $\left\{\widetilde{U}_{1}^{(l)}\left(x_{0}\right)\right\}_{l \in \mathscr{L}}$ следует (см. [21]), что семейство о.ф. $\left\{\widetilde{U}_{1}^{(l)}(z)\right\}_{l \in \mathscr{L}}$ равномерно сходится на компактах $K \subset \Pi_{-} \backslash$ $\left\{\mathscr{Z}^{(\infty)} \cup \overline{\mathscr{Z}}(\infty)\right\}$ к о.ф̆. $U_{1}^{(\infty)}(z)$.

Аналогичньм образом, используя $(37),(8)$ и (10), доказываем равномерную сходимость семейства $\left\{\widetilde{U}_{1}^{(l)}(z)\right\}_{l \in \mathscr{L}}$ на компактах $K \subset \mathbb{C}_{ \pm} \backslash\{\mathscr{Z}(\infty) \cup \overline{\mathscr{Z}}(\infty)\}$.

Объединяя полученные результаты, приходим к вьводу о равномерной сходимости семейства $\left\{\widetilde{U}_{1}^{(l)}(z)\right\}_{l \in \mathscr{L}}$ на компактах $K \subset \mathbb{C} \backslash\left\{\mathbb{R}_{+} \cup \mathscr{Z}(\infty) \cup \overline{\mathscr{Z}}(\infty)\right\}$. Отсюда следует голоморфность о.ф. $U_{1}^{(\infty)}(z)$ при $z \in \mathbb{C} \backslash\left\{\mathbb{R}_{+} \cup \mathscr{Z}(\infty) \cup \overline{\mathscr{Z}}(\infty)\right\}$.

Доказано первое из соотношений (56). Остальные соотношения в (56) доказываются аналогичным образом.

ТЕОРЕМА 7. Пусть дано упорядоченное семейство вполне неопределен-

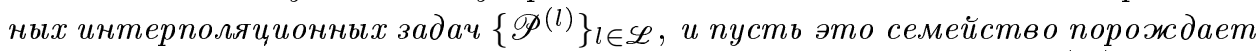
вполне неопределенную предельную интерполяционную задачу $\mathscr{P}(\infty)$.

Eсли о.ф. $s \in \mathscr{F}^{(\infty)}$, а о.ф. $U_{1}^{(\infty)^{-1}}$ и $U_{2}^{(\infty)^{-1}}$ определены в $(56)$, то выполнена система ОМН В. П. Потапова $\left(\forall z \in \mathbb{C}_{ \pm} \backslash\{\mathscr{Z}(\infty) \cup \overline{\mathscr{Z}}(\infty)\}\right)$

$$
\left[I \quad \bar{z}^{r-1} s^{*}(z)\right] \frac{U_{r}^{(\infty)^{-1}}(z) J U_{r}^{(\infty)^{-1}}(z)}{i(\bar{z}-z)}\left[\begin{array}{c}
I \\
z^{r-1} s(z)
\end{array}\right] \geqslant 0_{\mathscr{H}}, \quad r=1,2 .
$$

Наоборот, если для некоторой о.ф. $s \in \mathscr{S}$ выполняется система ОМН (57), $m o s \in \mathscr{F}(\infty)$. 
ДокаЗАТЕЛЬСтво. Пусть $s \in \mathscr{F}(\infty)$. Тогда для всех $l \in \mathscr{L}$ о.ф.. $s \in \mathscr{F}^{(l)}$ и вьполнена система ОМН $\left(\forall z \in \mathbb{C}_{ \pm} \backslash\{\mathscr{Z}(\infty) \cup \overline{\mathscr{Z}}(\infty)\}\right)$

$$
\left[I \quad \bar{z}^{r-1} s^{*}(z)\right] \frac{\widetilde{U}_{r}^{(l)^{-1^{*}}}(z) J \widetilde{U}_{r}^{(l)^{-1}}(z)}{i(\bar{z}-z)}\left[\begin{array}{c}
I \\
z^{r-1} s(z)
\end{array}\right] \geqslant 0_{\mathscr{H}}, \quad r=1,2 .
$$

Переходя к пределу $l \rightarrow A$, получаем неравенства (57).

Наоборот, пусть о.ф. $s \in \mathscr{S}$ и удовлетворяет системе ОМН (57). Зафиксируем некоторьй индекс $l \in \mathscr{L}$, и пусть индекс $k>l$. В силу (37) имеем $(r=1,2)$

$$
\frac{\left.\widetilde{U}_{r}^{(l)^{-1^{*}}}(z) J \widetilde{U}_{r}^{(l)}\right)^{-1}(z)}{i(\bar{z}-z)} \geqslant \frac{\widetilde{U}_{r}^{(k)^{-1^{*}}}(z) J \widetilde{U}_{r}^{(k)^{-1}}(z)}{i(\bar{z}-z)}, \quad z \in \mathbb{C}_{+} \backslash\left\{\mathscr{Z}^{(\infty)} \cup \overline{\mathscr{Z}}(\infty)\right\} .
$$

Переходя в этом неравенстве к пределу при $k \rightarrow A$, получаем $(r=1,2)$

$$
\frac{\left.\widetilde{U}_{r}^{(l)^{-1^{*}}}(z) J \widetilde{U}_{r}^{(l)}\right)^{-1}(z)}{i(\bar{z}-z)} \geqslant \frac{U_{r}^{(\infty)^{-1^{*}}}(z) J U_{r}^{(\infty)^{-1}}(z)}{i(\bar{z}-z)}, \quad z \in \mathbb{C}_{+} \backslash\left\{\mathscr{Z}^{(\infty)} \cup \overline{\mathscr{Z}}^{(\infty)}\right\}
$$

Умножим это неравенство слева на $\left[I \bar{z}^{r-1} s^{*}(z)\right]$, а справа - на сопряженный оператор. С учетом (57) получим $(r=1,2)$

$\left[I \quad \bar{z}^{r-1} s^{*}(z)\right] \frac{\left.\widetilde{U}_{r}^{(l)^{-1^{*}}}(z) J \widetilde{U}_{r}^{(l)}\right)^{-1}(z)}{i(\bar{z}-z)}\left[\begin{array}{c}I \\ z^{r-1} s(z)\end{array}\right] \geqslant 0, \quad z \in \mathbb{C}_{+} \backslash\{\mathscr{Z}(\infty) \cup \overline{\mathscr{Z}}(\infty)\}$

Таким образом, $s \in \mathscr{F}^{(l)}$. В силу произвольности $l$ имеем $s \in \mathscr{F}(\infty)$.

ТЕОРема 8. Пусть дано упорядоченное семейство вполне неопределенных интерполяционных задач $\left\{\mathscr{P}^{(l)}\right\}_{l \in \mathscr{L}}$, и пусть это семейство порождает вполне неопределенную предельную интерполяционную задачу $\mathscr{P}(\infty)$. И пусть, далее, о.ф. $U_{1}^{(\infty)}: \mathbb{C} \backslash\left\{\mathbb{R}_{+} \cup \mathscr{Z}(\infty) \cup \overline{\mathscr{Z}}(\infty)\right\} \rightarrow \mathscr{H} \oplus \mathscr{H}$ определена в $(57)$ и ее естественное матричное представление имеет вид

$$
U_{1}^{(\infty)}(z)=\left[\begin{array}{ll}
\alpha^{(\infty)}(z) & \beta^{(\infty)}(z) \\
\gamma^{(\infty)}(z) & \delta^{(\infty)}(z)
\end{array}\right]
$$

Тогда формула

$$
s(z)=\left\{\gamma^{(\infty)}(z) p(z)+\delta^{(\infty)}(z) q(z)\right\} \cdot\left\{\alpha^{(\infty)}(z) p(z)+\beta^{(\infty)}(z) q(z)\right\}^{-1}
$$

устанавливает биективное соответствие между $\mathscr{F}^{(\infty)}$ и $\mathscr{S}^{(\infty)}$.

Доказательство этой теоремы опирается на систему ОМН (57) и проводится по той же схеме, что и доказательство аналогичных утверждений в [20], [21]. 


\section{§7. Примеры}

7.1. Задача Неванлинны-Пика в классе Стилтьеса. В задаче Неванлинны-Пика задана бесконечная последовательность попарно различных комплексных чисел $z_{1}, z_{2}, \ldots, z_{k}, \ldots, z_{j} \neq \bar{z}_{k}, j, k \in \mathbb{N}$, и бесконечная последовательность операторов $s_{1}, s_{2}, \ldots, s_{k}, \ldots$, действующих в пространстве в $\mathscr{H}$. Требуется описать множество о.ф. $s: \mathbb{C} \backslash \mathbb{R}_{+} \rightarrow\{\mathscr{H}\}$ таких, что

$$
s\left(z_{j}\right)=s_{j} \quad \forall j \in \mathbb{N}, \quad s \in \mathscr{S} .
$$

Нас будет интересовать неопределенный случай, когда задача (59) имеет бесконечно много решений. Поэтому мы считаем, что выполнено необходимое условие неопределенности - предельные точки последовательности $\left\{z_{j}\right\}_{j \in \mathbb{N}}$ принадлежат $\mathbb{R}_{+} \cup\{\infty\}$.

Вместе с задачей (59) с бесконечным числом узлов интерполяции будем рассматривать и усеченные задачи Неванлинны-Пика. В таких задачах фиксируется число $k \in \mathbb{N}$ и требуется описать множество о.ф. $s: \mathbb{C} \backslash \mathbb{R}_{+} \rightarrow\{\mathscr{H}\}$ таких, что

$$
s\left(z_{j}\right)=s_{j}, \quad 1 \leqslant j \leqslant k, \quad s \in \mathscr{S} .
$$

Покажем, что усеченную задачу (60) можно рассматривать как обобщенную интерполяционную задачу стилтьесовского типа. В качестве масштабных пространств выберем пространства

$$
\mathscr{G}_{1}^{(k)}=\mathscr{G}_{2}^{(k)}=\underbrace{\mathscr{H} \oplus \mathscr{H} \oplus \cdots \oplus \mathscr{H}}_{k \text { слагаемых }}, \mathscr{H} .
$$

Операторы $K_{1}^{(k)}, L_{1}^{(k)}, v_{1}^{(k)}, K_{2}^{(k)}, L_{2}^{(k)}, u_{2}^{(k)}$, участвующие в задаче (2), зададим естественными матричными представлениями

$$
\begin{aligned}
L_{1}^{(k)} & =\operatorname{diag}\left\{I_{\mathscr{H}}, \ldots, I \mathscr{H}\right\} \in\left\{\mathscr{G}_{1}^{(k)}\right\}, \quad v_{1}^{(k)}=\operatorname{col}\left\{I_{\mathscr{H}}, \ldots, I \mathscr{H}\right\} \in\left\{\mathscr{H}, G_{1}^{(k)}\right\}, \\
L_{2}^{(k)} & =\operatorname{diag}\left\{z_{1}^{-1} I_{\mathscr{H}}, \ldots, z_{k}^{-1} I_{\mathscr{H}}\right\} \in\left\{\mathscr{G}_{2}^{(k)}\right\} \\
u_{2}^{(k)} & =L_{2}^{(k)^{-1}} \cdot \operatorname{col}\left\{s_{1}, \ldots, s_{k}\right\} \in\left\{\mathscr{H}, G_{2}\right\} . \\
K_{r}^{(k)} & =L_{2}^{(k)^{-1}} \cdot\left\{\frac{z_{i}^{r-1} s_{i}-\bar{z}_{j}^{r-1} s_{j}^{*}}{z_{i}-\bar{z}_{j}}\right\}_{i, j=1, \ldots, k} \cdot L_{2}^{(k)^{-1^{*}}} \in\left\{G_{r}\right\}, \quad r=1,2 .
\end{aligned}
$$

Очевидно, что выполнено ОТ (1). В [7] показано, что необходимым и достаточным условием разрешимости задачи (60) являются неравенства $K_{1}^{(k)} \geqslant 0, K_{2}^{(k)} \geqslant 0$. Более того (см. [7]), о.ф. $s \in \mathscr{S}$ является решением усеченной задачи (60) тогда и только тогда, когда она удовлетворяет системе ОМН (7). Таким образом, множество решений интерполяционной задачи (60) совпадает с множеством решений следуюшей интерполяционной задачи стилтьесовского типа:

$$
\mathscr{P}^{(k)}=\left\{K_{1}^{(k)}, L_{1}^{(k)}, v_{1}^{(k)}, K_{2}^{(k)}, L_{2}^{(k)}, u_{2}^{(k)}\right\} .
$$


Условиями полной неопределенности являются условия $K_{1}^{(k)}>0, K_{2}^{(k)}>0$. Легко видеть, что при этом все остальные условия в (8) автоматически выполнены, т.е. задача (61) является вполне неопределенной.

Пусть при всех $k \in \mathbb{N}$ интерполяционные задачи (61) являются вполне неопределенными. Покажем, что интерполяционные задачи (61) являются упорядоченньм семейством. Действительно, пусть $k$ и $l$ - произвольные натуральные числа и пусть, для определенности, $k>l$. Тогда пространства $\mathscr{G}_{r}^{(k)}$ можно представить в виде ортогональной суммы $\mathscr{G}_{r}^{(k)}=\widetilde{\mathscr{G}}_{r}^{(k)} \oplus \widetilde{\mathscr{G}}_{r}^{(k)}$, где

$$
\tilde{\mathscr{G}}_{r}^{(k)}=\underbrace{\mathscr{H} \oplus \ldots \oplus \mathscr{H}}_{l} \oplus \underbrace{0 \oplus \cdots \oplus 0}_{(k-l)}, \quad \widehat{\mathscr{G}}_{r}^{(k)}=\underbrace{0 \oplus \cdots \oplus 0}_{l} \oplus \underbrace{\mathscr{H} \oplus \cdots \oplus \mathscr{H}}_{(k-l)} .
$$

Легко видеть, что в естественных матричных представлениях операторы $\widetilde{P}_{r}^{(k)}$ имеют вид

$$
\widetilde{P}_{r}^{(k)}=\operatorname{diag}\{\underbrace{I_{\mathscr{H}}, \ldots, I_{\mathscr{H}}}_{l}, \underbrace{0 \mathscr{H}, \ldots, 0 \mathscr{H}}_{(k-l)}\} \in\left\{\mathscr{G}_{1}^{(k)}\right\} .
$$

Отсюда следует, что выполнены условия (32). Рассмотрим интерполяционную задачу $\widetilde{\mathscr{P}}^{(k)}$, которая является сужением интерполяционной задачи $\mathscr{P}(k)$ на подпространства $\widetilde{\mathscr{G}}_{r}^{(k)}$. Легко видеть, что интерполяционная задача $\mathscr{P}(l)$ унитарно эквивалентна интерполяционной задаче $\widetilde{\mathscr{P}}^{(k)}$. Таким образом, семейство интерполяционных задач (61) упорядочено. Поэтому теорема 5 задает критерий неопределенности предельной интерполяционной задачи (59), а формула (58) дает описание всех решений интерполящионной задачи (59) во вполне неопределенном случае.

7.2. Проблема моментов Стилтьеса. В проблеме моментов Стилтьеса по заданной последовательности операторов $s_{0}, \ldots, s_{k}, \ldots \in\{\mathscr{H}\}_{H}$ требуется описать множество монотонно возрастающих о.ф. $\sigma: \mathbb{R}_{+} \rightarrow\{\mathscr{H}\}_{H}$ таких, что

$$
s_{j}=\int_{0}^{+\infty} t^{j} d \sigma(t) \quad \forall j \in \mathbb{N} \cup\{0\}
$$

Можем считать, не изменяя значений интегралов, что о.ф. $\sigma$ удовлетворяет следующим условиям нормировки: $\sigma(t)$ непрерывна слева при $t \in(0,+\infty)$ и $\sigma(0)=0$. Множество нормированных решений $\sigma$ проблемы моментов (62) обозначим символом $\mathscr{M}^{(\infty)}$. С каждой $\sigma \in \mathscr{M}^{(\infty)}$ свяжем о.ф.

$$
s(z)=\int_{0}^{+\infty} \frac{d \sigma(t)}{t-z}
$$

Оператор-функция $s$ определена и голоморфна в $\mathbb{C} \backslash \mathbb{R}_{+}$и называется ассоииированной с проблемой моментов (62). Множество о.ф. $s$, ассоциированных с проблемой $(62)$, обозначим символом $\mathscr{F}(\infty)$. Из формулы обращения Стилтьеса следует, что соответствие, устанавливаемое между $\mathscr{F}^{(\infty)}$ и $\mathscr{M}^{(\infty)}$ формулой $(63)$, является взаимно однозначным. Поэтому вместо описания множества $\mathscr{M}^{(\infty)}$ мы можем ограничиться описанием множества $\mathscr{F}(\infty)$.

Вместе с бесконечной проблемой моментов (62) будем рассматривать и усеченные проблемы моментов Стилтьеса. В таких проблемах фиксируется число 
$k \in \mathbb{N} \cup\{0\}$ и требуется описать все нормированные монотонно возрастаюшие о.ф. $\sigma: \mathbb{R}_{+} \rightarrow\{\mathscr{H}\}_{H}$ и операторы $M \in\{\mathscr{H}\}_{\geqslant}$такие, что

$$
s_{j}=\int_{0}^{+\infty} t^{j} d \sigma(t), \quad 0 \leqslant j \leqslant k-1, \quad s_{k}=\int_{0}^{+\infty} t^{k} d \sigma(t)+M .
$$

При $k=0$ требуем выполнения только второго условия в (64).

Проблема моментов (64) называется $k$-й усеченной проблемой моментов Стилтьеса, а множество ее решений $\sigma$ обозначается символом $\mathscr{M}^{(k)}$. Как и в случае проблемы моментов (62), с каждой $\sigma \in \mathscr{M}^{(k)}$ свяжем ассоциированную о.ф. $s$ вида (63). Множество всех о.ф. $s$, ассоциированных с проблемой (64), обозначим символом $\mathscr{F}^{(k)}$.

Покажем, что задачу описания ассоциированных о.ф. можно рассматривать как обобщенную интерполяционную задачу стилтьесовского типа. Рассмотрим отдельно случай четного и нечетного числа моментов.

Пусть $k=2 n, n \in \mathbb{N}$. В этом случае в качестве масштабных пространств выберем пространства

$$
\mathscr{G}_{1}^{(k)}=\underbrace{\mathscr{H} \oplus \mathscr{H} \oplus \cdots \oplus \mathscr{H}}_{n+1}, \quad \mathscr{G}_{2}^{(k)}=\underbrace{\mathscr{H} \oplus \mathscr{H} \oplus \cdots \oplus \mathscr{H}}_{n}, \quad \mathscr{H} .
$$

Операторы $K_{1}^{(k)}, L_{1}^{(k)}, v_{1}^{(k)}, K_{2}^{(k)}, L_{2}^{(k)}, u_{2}^{(k)}$, участвующие в задаче (2), зададим естественными матричными представлениями

$$
\begin{aligned}
& K_{1}^{(k)}=\left\{s_{j+k}\right\}_{j, k=0}^{n}, \quad L_{1}^{(k)}= {\left[\begin{array}{ccc}
I_{\mathscr{H}} & \ldots & 0_{\mathscr{H}} \\
\vdots & \ddots & \vdots \\
0 \mathscr{H} & \ldots & I_{\mathscr{H}} \\
0 \mathscr{H} & \ldots & 0_{\mathscr{H}}
\end{array}\right], v_{1}^{(k)}=\left[\begin{array}{c}
I_{\mathscr{H}} \\
0_{\mathscr{H}} \\
\vdots \\
0_{\mathscr{H}}
\end{array}\right], } \\
& K_{2}^{(k)}=\left\{s_{j+k+1}\right\}_{j, k=0}^{n-1}, \quad L_{2}^{(k)}=\left[\begin{array}{ccc}
0 \mathscr{H} & \ldots & 0 \\
I_{\mathscr{H}} & \ldots & 0 \\
\vdots & \ddots & \vdots \\
0 \mathscr{H} \\
0_{\mathscr{H}} & \ldots & I_{\mathscr{H}}
\end{array}\right], u_{2}^{(k)}=-\left[\begin{array}{c}
s_{0} \\
s_{1} \\
\vdots \\
s_{n-1}
\end{array}\right] .
\end{aligned}
$$

Пусть $k=2 n+1, n \in \mathbb{N} \cup\{0\}$. В этом случае в качестве масштабных пространств выберем пространства

$$
\varphi_{1}^{(k)}=\mathscr{G}_{2}^{(k)}=\underbrace{\mathscr{H} \oplus \mathscr{H} \oplus \cdots \oplus \mathscr{H}}_{n+1}, \mathscr{H} .
$$

Операторы $K_{1}^{(k)}, L_{1}^{(k)}, v_{1}^{(k)}, K_{2}^{(k)}, L_{2}^{(k)}, u_{2}^{(k)}$, участвующие в задаче (2), зададим естественными матричными представлениями

$$
\begin{aligned}
& K_{1}^{(k)}=\left\{s_{j+k}\right\}_{j, k=0}^{n}, \quad L_{1}^{(k)}=\left[\begin{array}{cccc}
I_{\mathscr{H}} & 0_{\mathscr{H}} & \ldots & 0_{\mathscr{H}} \\
0_{\mathscr{H}} & I_{\mathscr{H}} & \ldots & 0_{\mathscr{H}} \\
\vdots & \vdots & \ddots & \vdots \\
0_{\mathscr{H}} & 0_{\mathscr{H}} & \ldots & I_{\mathscr{H}}
\end{array}\right], \quad v_{1}^{(k)}=\left[\begin{array}{c}
I_{\mathscr{H}} \\
0_{\mathscr{H}} \\
\vdots \\
0_{\mathscr{H}}
\end{array}\right], \\
& K_{2}^{(k)}=\left\{s_{j+k+1}\right\}_{j, k=0}^{n}, \quad L_{2}^{(k)}=\left[\begin{array}{cccc}
0 \mathscr{H} & \ldots & 0_{\mathscr{H}} & 0_{\mathscr{H}} \\
I_{\mathscr{H}} & \ldots & 0_{\mathscr{H}} & 0_{\mathscr{H}} \\
\vdots & \ddots & \vdots & \vdots \\
0_{\mathscr{H}} & \ldots & I_{\mathscr{H}} & 0_{\mathscr{H}}
\end{array}\right], u_{2}^{(k)}=-\left[\begin{array}{c}
s_{0} \\
s_{1} \\
\vdots \\
s_{n}
\end{array}\right] \text {. }
\end{aligned}
$$


Непосредственно проверяем, что определенные выше операторы при $k=2 n, n \in \mathbb{N}$, и при $k=2 n+1, n \in \mathbb{N} \cup\{0\}$, удовлетворяют ОТ (1). В [7] показано, что необходимым и достаточным условием разрешимости задачи (64) являются неравенства $K_{1}^{(k)} \geqslant 0, K_{2}^{(k)} \geqslant 0$. Более того (см. [7]), о.ф.. $s \in \mathscr{F}^{(k)}$ тогда и только тогда, когда она удовлетворяет системе $\mathrm{OMH}(7)$. Таким образом, множество $\mathscr{F}^{(k)}$ совпадает со множеством решений следующей интерполяционной задачи стилтьесовского типа:

$$
\mathscr{P}^{(k)}=\left\{K_{1}^{(k)}, L_{1}^{(k)}, v_{1}^{(k)}, K_{2}^{(k)}, L_{2}^{(k)}, u_{2}^{(k)}\right\} .
$$

Условиями полной неопределенности являются условия $K_{1}^{(k)}>0, K_{2}^{(k)}>0$. Легко видеть, что при этом все остальные условия в (8) автоматически выполнены, т.е. задача (65) является вполне неопределенной. Будем считать, что задачи (65) являются вполне неопределенными при всех $k$.

Как и в случае задачи Неванлинны-Пика, убеждаемся в том, что интерполяционные задачи (65) являются упорядоченным семейством. Поэтому теорема 5 задает критерий неопределенности предельной интерполяционной задачи (62), а формула (58) дает описание всех решений предельной интерполяционной задачи (62) во вполне неопределенном случае.

\section{Список литературы}

1. Нудельман А. А. Об одном обобщении классических интерполяционных задач // Докл. AH CCCP. 1981. T. 256. № 4. C. 790-793.

2. Иванченко Т. С., Сахнович Л. А. Операторный подход к схеме В.П. Потапова исследования интерполяционных задач // Укр. матем. журн. 1987. Т. 39. № 5. С. 573-578.

3. Качнельсон В.Я., Хейфеч А.Я., Юдицкий П. М. Абстрактная интерполяционная проблема и теория расширений изометрических операторов // Операторы в функциональных пространствах и вопросы теории функций. Сборник научных трудов. Киев: Наукова думка, 1987. С. 83-96.

4. Kheifets A. Ya., Yuditskii P. M. Ah analysis and extension of V.P. Potapov approach to interpolation problems with applications to the generalized bi-tangential Schur-NevanlinnaPick problem and J-inner-outer factorization // Oper. Theory Adv. Appl. 1994. V. 72. P. $133-161$.

5. Ivanchenko T.S., Sakhnovich L. A. An operator approach to the Potapov scheme for the solution of interpolation problems // Oper. Theory Adv. Appl. 1994. V. 72. P. 48-86.

6. Dyukarev $Y u$. M. Integral representations of a pair of nonnegative operators and interpolation problems in the Stieltjes class // Oper. Theory Adv. Appl. 1997. V. 95. P. 165-184.

7. Дюкарев Ю. М. Обшая схема решения интерполяционных задач в классе Стилтьеса, основанная на согласованных интегральных представлениях пар неотрицательных операторов. I // Матем. физика, анализ, геом. 1999. Т. 6. №1/2. С. 30-54.

8. Bolotnikov $V$., Sakhnovich $L$. On an operator approach to interpolation problems for Stieltjes fanctions // Integral Equations Operator Theory. 1999. V. 35. P. 423-470.

9. Stieltjes T. Recherches sur les fractions continues // Ann. Fac. Sci. Toulouse Math. (6). 1894. V. 8. P. 1-122; 1895. V. 9. P. 1-47.

10. Schur I. Über Potenzreihen, die im Innern des Einheitskreises beschränkt sind // J. Reine Angew. Math. 1917. V. 147. P. 205-232; 1918. V. 148. P. 122-145.

11. Hamburger $H$. Über eine Erweitung des Stieltjesschen Momentenproblem // Math. Ann. 1920. V. 81. P. 235-319; 1921. V. 82. P. 120-164; 1921. V. 82. P. 168-187.

12. Ковалишина И.В., Потапов В. П. Индефинитная метрика в проблеме Неванлинны-Пика // Докл. АН АрмССР. 1974. Т. 59. №1. С. 17-22.

13. Ковалишина И. В. Аналитическая теория одного класса интерполяционных задач // Изв. АН СССР. Сер. матем. 1983. Т. 47. №3. С. 455-497. 
14. Потапов В. П. К теории матричных кругов Вейля // Функциональный анализ и прикладная математика. Киев: Наукова думка, 1982. С. 113-121.

15. Потапов В. П. Дробно-линейные преобразования матриц // Исследования по теории операторов и их приложениям. Киев: Наукова думка, 1979. С. 75-97.

16. Крейн М. Г., Нудельман А. А. Проблема моментов Маркова и экстремальные задачи. М.: Наука, 1973.

17. Дюкарев Ю. М., Кацнельсон В.Э. Мультипликативные и аддитивные классы Стилтьеса аналитических матриц-функций и связанные с ними интерполяционные задачи // Теория функций, функц. анализ и их прилож. 1981. № 36. С. 13-27.

18. Дюкарев Ю. М., Чоке Риверо A.E. Степенная проблема моментов на компактном интервале // Матем. заметки. 2001. Т. 69. № 2. С. 200-213.

19. Simon $B$. The classical moment problem as a self-adjoint finite differense operator // Adv. Math. 1998. V. 137. P. 82-203.

20. Donoghue W. F. Monotone matrix functions and analytic continuation. Berlin: SpringerVerlag, 1974.

21. Орлов C.A. Гнездящиеся матричные круги, аналитически зависящие от параметра, и теоремы об инвариантности рангов радиусов предельных матричных кругов // Изв. АН СССР. Сер. матем. 1976. Т. 40. № 3. С. 593-644.

Харьковский национальньй университет, Украина

E-mail: Yury.M.Dyukarev@univer.kharkov.ua

Поступила в редакцию

03.06 .2003 